INRA Prod. Anim., 2017, 30 (4), 333-350

\section{La diversité des services rendus par les territoires d'élevage herbagers fournissant des produits de qualité dans des environnements préservés}

\author{
D. VOLLET', O. HUGUENIN-ELIE ${ }^{2}$, B. MARTIN ${ }^{3}$, B. DUMONT \\ ${ }^{1}$ Université Clermont Auvergne, AgroParisTech, INRA, Irstea, VetAgro Sup, \\ Territoires, 63000 Clermont-Ferrand, France \\ ${ }_{2}^{2}$ Agroscope, Production fourragère et systèmes herbagers, CH-8046 Zurich, Suisse \\ ${ }_{3}^{3}$ Université Clermont Auvergne, INRA, VetAgro Sup, UMR Herbivores, 63122, Saint-Genès-Champanelle, France
}

Courriel :dominique.vollet@irstea.fr

La fourniture de services à la société est un pilier important des activités d'élevage dans les environnements préservés, car celle-ci confère une image positive aux produits issus de ces activités. Néanmoins, ces élevages gèrent habituellement des milieux sensibles et accueillant différents types d'activités, où le risque d'impacts négatifs sur l'environnement ou d'antagonismes avec d'autres utilisations est élevé. Cet article vise à analyser les conditions cadres et la fourniture de services de quatre de ces territoires qui présentent d'importantes différences de contexte de production.

Les zones herbagères de moyenne densité animale représentent près de $20 \%$ de la SAU européenne. En France et en Suisse, elles sont essentiellement situées dans les massifs montagneux de moyenne ou haute altitude (essentiellement le Massif central, le massif Jurassien, les Alpes du nord et la partie occidentale des Pyrénées) et dans une moindre mesure en Basse Normandie (Hercule et al 2017, ce numéro). Elles sont le lieu où sont élaborés des produits de qualité dans des environnements plutôt préservés qui ont une forte attractivité pour les populations urbaines. Ces territoires d'élevage fournissent de multiples services de nature et d'ampleur très différente (dans cet article nous utilisons l'expression « bouquet de services »). Les différentes activités qu'accueillent ces territoires (élevage, tourisme, loisir) offrent l'opportunité de profiter de synergies entre activités (par exemple maintien de paysages ouverts par l'élevage et activités de loisir et vente des produits d'élevage). Cependant, l'élevage peut aussi entraver d'autres activités et porter atteinte à l'environnement dans ces milieux sensibles (on parle alors d'antagonismes, de dys-services, cf. Dumont et al 2017, ce numéro, pour une définition de l'ensemble de ces termes). Une gestion équilibrée de ces territoires nécessite donc de mieux comprendre les déterminants du bouquet de services qu'y rend l'élevage.
Nous portons ici notre analyse sur des territoires d'ampleur géographique et de contexte de production très différents : un massif montagneux ; le Massif central, une région administrative ; la FrancheComté, et une zone d'estive dans les Alpes suisses. Pour le Massif central, deux zones sont différenciées, les massifs du Cantal et de l'Aubrac, afin de mieux identifier les leviers de développement conjoints de plusieurs services. Ces territoires sont le lieu de production de fromages au lait de vache bénéficiant d'une Appellation d'Origine Protégée (AOP). La part de la transformation fermière est très variable : faible pour certaines (Cantal dans le Massif central) élevée voire exclusives pour une minorité (fromage d'alpage en Suisse), ce qui créée de la valeur ajoutée sur l'exploitation et des emplois au niveau local. Contrairement aux autres territoires étudiés, le Massif central possède par ailleurs un important troupeau bovin allaitant; les productions standards (en maigre ou en gras) ou label (pour la viande) coexistent avec les productions AOP. Les systèmes herbagers supports de ces productions sont caractérisés par une grande diversité de prairies permanentes qui constituent un important réservoir de biodiversité et contribuent à la typicité des fromages. Les productions de fromages AOP mettent en avant une production à base d'herbe dans leurs cahiers des charges. Elles sont prisées des consommateurs et valorisent au mieux les conditions climatiques liées à l'altitude, qui limitent le développement de l'ensilage de maïs. Ce cadre général étant posé, une première partie de l'article sera consacrée à la description des principaux processus biologiques sous-jacents aux services spécifiques rendus par ces systèmes herbagers. Dans les trois parties suivantes, nous décrivons de façon approfondie les spécificités du Massif central (Cantal et Laguiole), de la Franche-Comté, et des Alpes suisses. Les leviers d'action sont exposés dans une cinquième et dernière partie.

\section{1 / Les processus biologiques sous-jacents aux services rendus par les exploitations laitières herbagères}

\section{1 / Alimentation des vaches} laitières à l'herbe et qualité nutritionnelle et sensorielle des fromages

La composition de la ration des vaches laitières a un effet rapide et fort sur la composition du lait qui elle-même modifie les caractéristiques des fromages. En comparaison des rations à base d'ensilage de maïs, les rations à base d'herbe pâturée 
modifient le profil en acides gras du lait et des fromages dans un sens favorable du point de vue de leur valeur santé pour le consommateur (ANSES 2011). Le pâturage est à l'origine de matières grasses dont la proportion en acides gras saturés les plus délétères vis-à-vis des maladies cardiovasculaires (acides laurique, myristique et palmitique) sont nettement diminuées au profit d'acides gras insaturés considérés comme neutres (acide oléique) ou bénéfiques ( $\alpha$-linolénique, Coppa et al 2013). De plus, le rapport entre les acides gras oméga6/oméga-3 diminue nettement, ce qui est également favorable du point de vue de la valeur santé pour le consommateur. Les laits et les fromages sont aussi plus riches en vitamines liposolubles (vitamines A et E) et en antioxydants tels que les caroténoïdes et les composés phénoliques. Les foins et les ensilages d'herbe conduisent à des laits et des fromages qui présentent des caractéristiques intermédiaires entre le pâturage et l'ensilage de maïs.

Sur le plan organoleptique, le pâturage est à l'origine de fromages souvent plus appréciés par les consommateurs (Martin et al 2009) en raison de la coloration jaune de leur pâte (liée à la teneur élevée de leur lait en pigments caroténoïdes) et de leur texture nettement plus fondante et moins ferme (liée au point de fusion plus bas des matières grasses). Ils développent des flaveurs plus corsées et diversifiées, en comparaison des fromages issus de vaches nourries avec des régimes à base d'ensilage de maïs (Martin et al 2005). L'effet bénéfique du pâturage sur la flaveur des fromages est atténué lorsque le lait est pasteurisé. Les caractéristiques des fromages issus de rations à base de foin ou d'ensilage d'herbe sont proches (Verdier-Metz et al 2005) ; elles sont intermédiaires entre le pâturage et l'ensilage de maïs.

Par ailleurs, comparativement au pâturage de prairies temporaires, le pâturage de prairies permanentes diversifiées est à l'origine d'une augmentation dans le lait et le fromage de la teneur en acide $\alpha$-linolénique et d'une réduction de la teneur en acides gras saturés les plus délétères vis-à-vis des maladies cardiovasculaires, notamment l'acide palmitique (Ferlay et al 2013). Les fromages issus des prairies les plus diversifiées présentent également une palette d'arômes plus étendue et une flaveur globale moins intense que les fromages issus de prairies moins diversifiées. Aux dires de certains fromagers, le pâturage de prairies diversifiées conduit à des fromages qui s'affinent plus lentement, donc plus longtemps, permettant ainsi l'expression de leur potentiel aromatique (Martin et al 2005).

\section{2 / Conduite des prairies et bio- diversité}

Les exploitations laitières des territoires analysés dans ce chapitre comportent une grande diversité de prairies permanentes qui stockent autour de 60-80 t C/ha.an (Angers et al 2011, Carrère et al 2012) soit nettement plus que les prairies temporaires. Les légumineuses prairiales augment également le stockage du carbone organique dans le sol comparativement aux couverts de graminées pures (Lüscher et al 2014). La diversité floristique des prairies est fonction des conditions de milieu (température, humidité, nature et fertilité du sol) et des modes d'utilisation des couverts (fauche $v s$ pâture, intensité et période d'utilisation, fertilisation). L'hypothèse de «stress intermédiaire » illustrée par une courbe en cloche (Milchunas et al 1988) prédit que la diversité floristique des prairies sera maximale pour les niveaux médians des facteurs agronomiques, c'est-à-dire lorsque le chargement est allégé et la fertilisation ou le nombre de fauches réduits. Ceci a été observé dans différents réseaux de parcelles (Klimek et al 2007, Marini et al 2008).

Les courbes de Milchunas et al (1988) révèlent par ailleurs des interactions entre les facteurs du milieu (par exemple la fertilité du sol) et l'intensité d'utilisation des prairies. Ainsi, lorsqu'on réduit le chargement de prairies fertiles, les espèces « bonnes fourragères » telles que le ray-grass et le trèfle blanc régressent et sont remplacées par des espèces de moindre valeur nutritive (Loiseau et al 1998, Dumont et al 2011). L'espèce animale a un effet additionnel; les bovins ont un comportement de prélèvement moins sélectif que les ovins, et structurent plus fortement les couverts. Leur pâturage préférentiel des placettes d'herbe végétative de « bonne qualité » crée une mosaïque relativement stable de placettes rases et de zones de refus qui diversifient les habitats tout en maintenant la valeur nutritive de ce qui est prélevé (Dumont et al 2012). Dans les prairies peu fertiles, une réduction du niveau de chargement accroît la part relative des plantes à fleur (Dumont et al 2009), ce qui profite aux insectes phytophages, tels que les orthoptères (Marini et al 2008), et aux nectarivores tels que les papillons et les bourdons (Dumont et al 2009, Milberg et al 2016). Laisser temporairement au repos certaines parcelles permet ainsi de compenser pour partie les effets d'une utilisation intensive d'autres surfaces de l'exploitation (Franzen et Nilsson 2008). C'est le principe des surfaces de promotion de la biodiversité instaurées en Suisse au milieu des années 1990, et qui imposent une gestion environnementale sur $7 \%$ des parcelles d'une exploitation. Le choix des périodes de pâturage revêt aussi une importance majeure (Davis et al 2014). Ainsi, est-il possible de préserver la biodiversité prairiale tout en maintenant les niveaux de production animale en excluant une partie des parcelles du pâturage au pic de floraison (Ravetto Enri et al 2017). Au-delà de la conduite des prairies, la préservation des infrastructures paysagères telles que les haies, les bois et les mares contribue à la préservation de la biodiversité au sein des paysages agro-pastoraux (Buttler 2014). Les haies et les bosquets jouent alors un rôle de corridors écologiques qui relient entre eux des habitats vitaux pour une espèce.

\section{2 / Zones AOP de montagne dans le Massif central}

La dynamique des productions animales dans les zones AOP du Massif central se caractérise par une grande diversité de situations : coexistence sur certains territoires de productions standards et AOP, spécialisation d'autres territoires en productions AOP avec des niveaux de valorisation très variables de ces AOP. Pour cette raison, nous avons retenu de décrire deux filières très différentes du point de vue de leurs évolutions, les filières Cantal et Laguiole.

\section{1 / Une filière AOP Cantal faiblement rémunératrice pour les agriculteurs mais intensive en emplois indirects}

a) Des évolutions des cahiers des charges qui ont distendu le lien au territoire

La production de l'AOC Cantal a été marquée par des évolutions très contrastées de son lien au territoire (figure 1). Le premier texte de 1956 donnant naissance à l'AOC Cantal était d'une grande précision sur les facteurs naturels et anthropiques de l'aire de production (altitude de 600 à $1500 \mathrm{~m}$, nature volcanique des terrains, utilisation des races Salers et Aubrac uniquement, utilisation exclusive du pâturage, collecte effectuée deux fois par jour). Rapidement, des mutations profondes ont permis d'accroître la productivité et ont affecté le système de production : remplacement partiel des deux races initiales au profit de races spécialisées (holstein et montbéliarde), développement de l'ensilage de maïs, déplacement d'une partie de la production vers les zones non volcaniques et de faible altitude (Châtaigneraie cantalienne), industrialisation de la transformation avec adoption des techniques de thermisation et de pasteurisation du lait, réduction du temps de fabrication et d'affinage.

Ces transformations, qui distendent le lien au territoire, ont été entérinées par 
Figure 1. Frise temporelle de l'AOP Cantal.

- Décrets ministériels de 1980 et 1986 entérinant évolutions vers intensification production et liens plus lâches avec le territoire

- Développement troupeaux FFPN, montbéliardes, intensification production hors berceau initial dans la partie granitique (châtaigneraie)

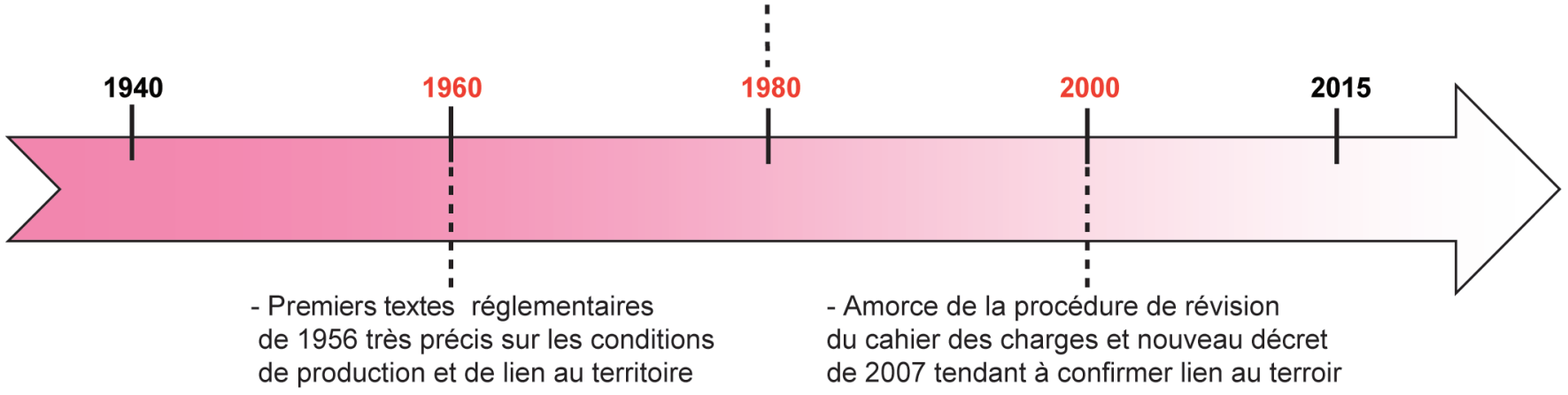

les décrets ministériels de 1980 et 1986 , sous la pression des entreprises les plus importantes. Le déplacement des demandes sociales vers des produits plus qualitatifs, plus respectueux de l'environnement et la faible rémunération des éleveurs locaux ont incité les acteurs de la filière à renforcer de nouveau les liens au territoire à partir des années 1990. Après de longues négociations, un accord est finalement trouvé en 2007 : des restrictions sont apportées dans le cahier des charges quant à la gestion des surfaces fourragères, l'alimentation du cheptel et les procédés technologiques. Cette réorientation reste cependant éloignée du décret initial de 1956 et présente un niveau d'exigence assez faible par rapport aux autres AOP, qui ne garantit pas nécessairement de rémunération supplémentaire aux producteurs (la différenciation du produit étant limitée).

\section{b) Une AOP marquée par une production importante}

Parmi les productions laitières du nord du Massif central, l'AOP Cantal se distingue par une production abondante (13 900 tonnes en 2014). La zone de production s'étend sur l'ensemble du Cantal plus quelques franges dans la Corrèze et le Puy de Dôme. Elle concerne près de 1300 producteurs.

L'utilisation d'herbe comme constituant principal des rations des vaches laitières a des effets bénéfiques sur la qualité sensorielle et nutritionnelle des fromages (Lucas et al 2006, Martin et al 2009 ). Cet effet est d'autant plus marqué que la proportion de prairies permanentes est importante dans ces zones. Il faut toutefois noter que dans le sud de la zone de production, l'ensilage de maïs tient toujours une place importante dans les systèmes fourragers. Par ailleurs, le Cantal, comme la majorité des fromages AOP du Massif central autorise l'uti- lisation d'herbe conservée sous forme d'ensilage.

c) Des services environnementaux très liés au chargement des prairies et en retrait en raison du développement de l'ensilage

Le développement des techniques d'ensilage d'herbe et d'enrubannage accompagnées d'une augmentation de la fertilisation azotée a permis d'avancer les dates de fauche d'au moins un mois, leurs effets s'ajoutant à ceux du changement climatique. L'exploitation des prairies a ainsi lieu bien avant la période de floraison de la plupart des espèces, ce qui réduit leur production de graine et à terme la diversité floristique du milieu (Carrère et al 2002). Ce mode d'exploitation perturbe les relations trophiques au sein des couverts et a un impact indirect sur les insectes nectarivores à qui manquent les ressources produites par les fleurs (Dumont et al 2009, Milberg et al 2016). La généralisation de telles techniques risque à terme d'entraîner non seulement une banalisation du paysage, avec la disparition progressive des prairies fleuries et colorées du début d'été, mais aussi une érosion de leur biodiversité.

Malgré ces menaces, la diversité des prairies permanentes reste élevée. Des observations de terrains complétées par des relevés phytosociologiques ont conduit à identifier 60 types de prairies permanentes ou semées pour l'ensemble des zones laitières AOP du Massif central (www.prairies-aoc.net). Vingttrois types sont couramment rencontrés (Carrère et al 2012) ; 19 d'entre eux sont des prairies permanentes qui stockent en moyenne $78 \mathrm{t}$ de matière organique dans les 10 premiers $\mathrm{cm}$ du sol (min : 56 , max : 122) et ont une richesse floristique moyenne de 33 espèces par parcelle (min : 18, max : 39). Les quatre types de prairies semées ne stockent en moyenne que $38 \mathrm{t}$ de matière organique dans les 10 premiers $\mathrm{cm}$ du sol et ont une richesse floristique moyenne de 25 espèces par parcelle. Ces 23 types constituent la base de la typologie des systèmes laitiers AOP du Massif central (Carrère et al 2012, Theau et al 2012). Chaque type est caractérisé par son potentiel de stockage du carbone (dix des 19 types de prairies permanentes ont une note élevée, 3 ou 4), son intérêt patrimonial botanique (entre 1 et 5 , huit types ayant la note 4 ou 5), la diversité de couleurs de fleurs (entre 1 et 4 , huit types ayant la note 3 ou 4), la capacité d'accueil des pollinisateurs et de la faune.

d) Mise en évidence de quelques synergies ou compromis entre services environnementaux et d'approvisionnement

L'idée de mieux valoriser la diversité des prairies permanentes du Massif central a émergé progressivement pour répondre aux attentes des filières fromagères $\mathrm{AOP}$, dont les cahiers des charges ont évolué au travers d'un renforcement du lien entre le produit et son milieu (voir par exemple le décret AOP Cantal du 8 mars 2007). Dans cet esprit, la typologie des prairies des systèmes laitiers AOP du Massif central fournit un outil qui permet d'évaluer les pratiques des éleveurs (Theau et al 2012). Elle met en évidence le large spectre des services écosystémiques fournis par les prairies permanentes d'Auvergne, et quantifie certains des compromis déjà évoqués, par exemple entre valeur nutritive et intérêt patrimonial des prairies. La souplesse d'exploitation des prairies est positivement corrélée à leur diversité floristique. La richesse aromatique des fromages augmente avec le stade d'exploitation de l'herbe et avec la diversité floristique du couvert (Graulet et al 2012) alors que les conduites plus intensives permettent d'obtenir des fromages avec 
une pâte plus jaune, prisés des consommateurs (Ferlay et al 2006).

À l'échelle du système d'exploitation, la diversité des modes d'utilisation des surfaces offre de la souplesse dans la gestion du système fourrager et augmente l'autonomie fourragère des exploitations laitières (Andrieu et al 2007). Des parcelles moins productives mais plus diversifiées se voient attribuer des fonctions spécifiques dans le système fourrager, et permettent de concilier des objectifs de production et de conservation de la biodiversité. Ces réflexions se prolongent dans le cadre du projet CASDAR-ATOUS initié en 2014. Son objectif reste de favoriser l'autonomie fourragère des éleveurs laitiers engagés en production fromagère $\mathrm{AOP}$ tout en rendant compte de la diversité des services écosystémiques fournis par les prairies (Carrère et al 2015). Dix descripteurs fourragers (\% de lait produit sans concentrés...) et onze descripteurs des services écosystémiques (potentiel aromatique des fromages, accueil des pollinisateurs, stockage du carbone, couleurs des fleurs, longueur des chemins de randonnée...) ont été sélectionnés avec les conseillers agricoles. Ils permettent de cartographier la diversité des prairies qui sous-tend les bouquets de services. Les accompagnements mis en œuvre offrent un mode d'appropriation collectif de la notion de services écosystémiques par les éleveurs en lien avec l'enjeu d'autonomie fourragère.

\section{e) Un effet important sur la vitalité locale}

Les exploitations du département du Cantal (au cour de la zone de production de l'AOP) peinent à dégager des valeurs ajoutées supplémentaires. Seulement $36 \%$ des exploitations du Cantal dégagent un revenu par actif supérieur au SMIC (contre $79 \%$ dans le Doubs d'après l'indicateur assiette de cotisations MSA; Dervillé, 2012). Cette situation s'explique par le fait que la production d'une grande partie des appellations (notamment du Cantal) relève d'une gouvernance plus sectorielle que territoriale. Elle est en quelque sorte « privatisée " soit par des industriels, soit par des stratégies individuelles de différenciation (Dervillé 2012). L'importance de la production laitière permet le maintien de structures de transformation, qui occupent une part importante de l'emploi local : en 2014, plus de six salariés sur 10 employés dans les industries agroalimentaires travaillaient dans les industries laitières des bassins de Saint-Flour et de Mauriac (INSEE Analyses 2015).

Les principaux impacts positifs ou négatifs du système AOP Cantal représentés dans la figure 2 et le tableau 1 selon le formalisme de la " grange " (Duru et al 2017, ce numéro) sont les suivants :

i) Une production massive de produits de qualité qui s'appuie sur l'existence d'un cahier des charges liant de manière assez limitée la production au territoire ;

ii) une rémunération relativement faible des emplois agricoles en raison d'un mode de gouvernance sectoriel ;

iii) la présence de nombreux emplois dans la transformation agroalimentaire ;

iv) des services environnementaux d'ampleur et de natures différentes selon les pratiques, mais plutôt en retrait en raison de la possibilité d'ensilage.

\section{2 / Une gouvernance territoriale de la filière AOP Laguiole}

a) Une AOP fromagère qui s'appuie sur une organisation efficace

L'Aubrac se caractérise par des organisations relativement fortes héritées des années 1960-1970 : coopérative Jeune Montagne dès 1961, CEMAC, CDANA ${ }^{1}$, coopérative d'élevage du Massif central. Elles correspondent à l'émergence d'un mouvement syndicaliste issu de la Jeunesse Agricole Chrétienne. Celui-ci s'est investi dans l'encadrement professionnel

Tableau 1. Effets positifs et négatifs associés aux systèmes d'élevage de l'AOP Cantal.

\begin{tabular}{|c|c|c|}
\hline Interfaces étudiées & Effets positifs & Effets négatifs \\
\hline Intrants & - Autonomie fourragère élevée & $\begin{array}{l}\text { - Effets délocalisés de la production } \\
\text { d'aliments concentrés } \\
\text { - Fertilisation minérale des prairies } \\
\text { - Maïs ensilage }\end{array}$ \\
\hline Travail et emploi & $\begin{array}{l}\text { - Maintien du tissu rural } \\
\text { - Nombreux emplois dans les industries } \\
\text { de transformation }\end{array}$ & $\begin{array}{l}\text { - Faible rémunération du travail agricole liée } \\
\text { à une gouvernance sectorielle }\end{array}$ \\
\hline Marchés & - Production massive de produits de qualité & $\begin{array}{l}\text { - Valorisation économique faible à moyenne } \\
\text { (transformation du lait pour partie hors zone) } \\
\text { - Saisonnalité de la production }\end{array}$ \\
\hline $\begin{array}{l}\text { Effets sociaux } \\
\text { et culturels }\end{array}$ & $\begin{array}{l}\text { - Esthétique du paysage, identité culturelle } \\
\text { - Patrimoine gastronomique et culturel } \\
\text { - Préservation savoir-faire locaux } \\
\text { - Qualité nutritionnelle des produits }\end{array}$ & \\
\hline $\begin{array}{l}\text { Environnement } \\
\text { et climat }\end{array}$ & $\begin{array}{l}\text { - Biodiversité (prairies permanentes, habitats } \\
\text { remarquables, races) } \\
\text { - Séquestration du carbone } \\
\text { - Qualité des eaux }\end{array}$ & $\begin{array}{l}\text { - Émissions élevées de } \mathrm{CH}_{4} \text { par } \mathrm{kg} \text { de } \\
\text { produit } \\
\text { - Ensilage de maïs en concurrence de } \\
\text { l'alimentation à l'herbe } \\
\text { - Sensibilité aux aléas climatiques et aux } \\
\text { ravageurs }\end{array}$ \\
\hline
\end{tabular}

${ }^{1}$ Comité de Développement Agricole du Nord-Aveyron. 
Figure 2. Synthèse des services et impacts rendus par le système AOP Cantal.

Comme décrit par Duru et al (2017), le pentagone central représente un territoire d'élevage avec ses éléments paysagers (haies) et les activités agro-industrielles sur lesquelles s'appuie l'élevage. Celui-ci est décrit par ses caractéristiques : espèces et densité animales, modes d'alimentation (animal vert : pâturage et fourrages, ocre : aliments concentrés dont l'ensilage de maïs). Le mode d'usage des terres est représenté par un parcellaire à deux nuances de vert pour symboliser la diversité des prairies permanentes et la présence de temporaires, et à deux nuances de jaune pour représenter la diversité des rotations culturales. Au sein du territoire, les systèmes d'élevage interagissent avec cinq interfaces. Les pictogrammes explicités ci-dessous symbolisent les éléments concernés par ces interfaces. La nature et l'ampleur des effets sont représentées par une flèche sortante plus ou moins large et dont la couleur indique que ceux-ci sont positifs (flèche verte), négatifs (rouge) ou mitigés (hachures); dans ce cas, l'effet dominant borde la flèche. Sur l'interface travail et emploi, nous distinguons les emplois directs en élevage des emplois indirects (IAA, tourisme, flèche vers personnage blanc). Les flèches entrantes indiquent l'utilisation d'intrants exogènes (qui symbolisent les effets délocalisés de l'élevage), ou (sur l'interface environnement et climat) que l'élevage bénéficie de services intrants (flèche verte) ou subit la pression de facteurs environnementaux (prédation, sécheresse... ; flèche rouge).

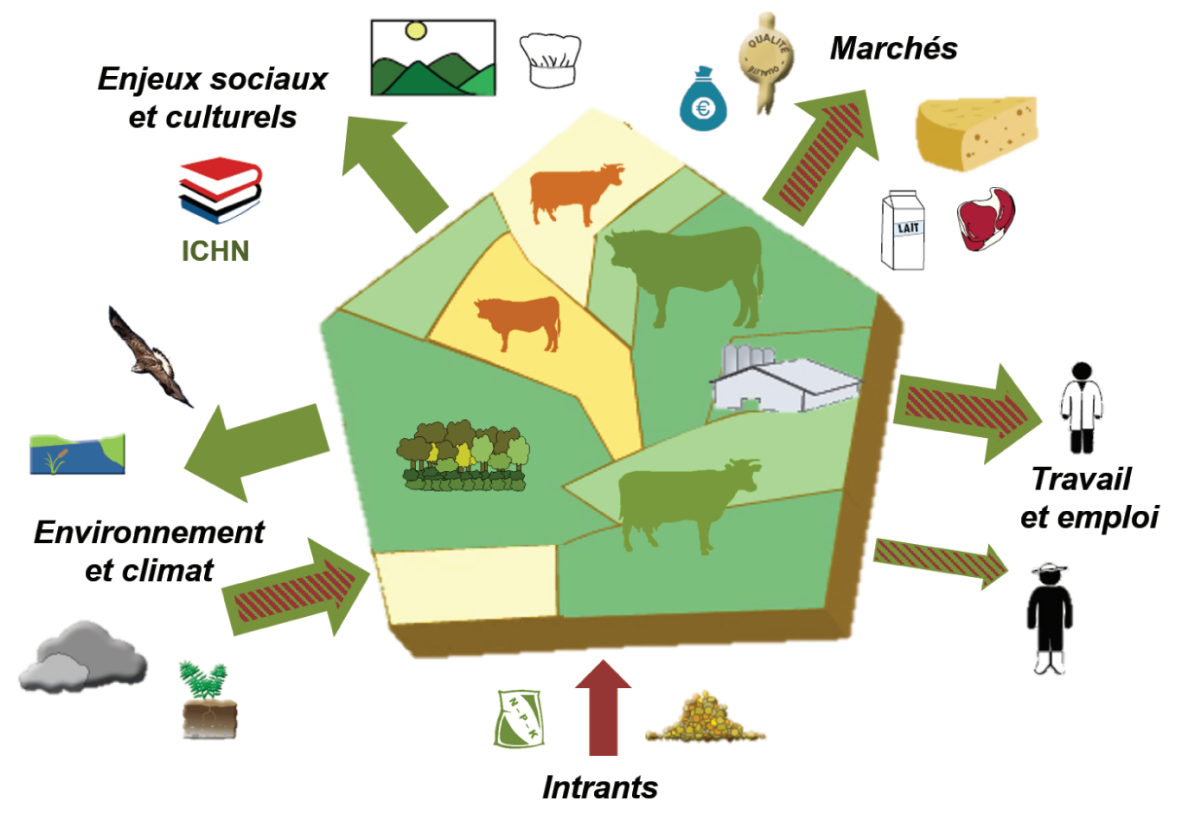

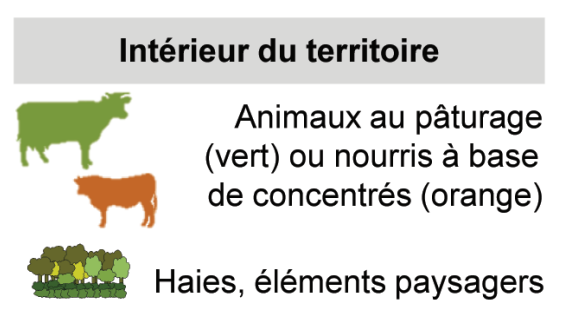

Environnement et climat

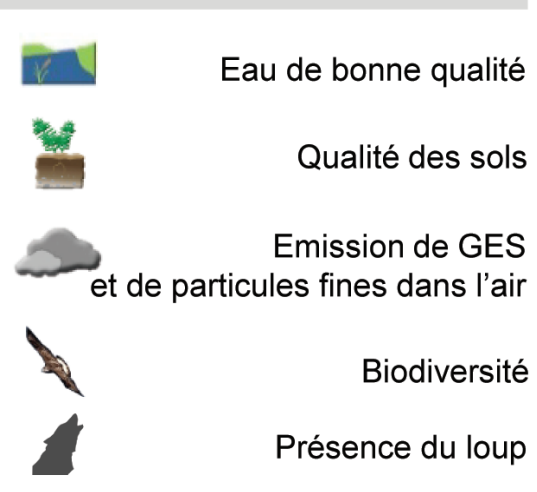

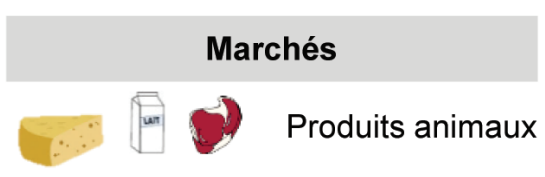

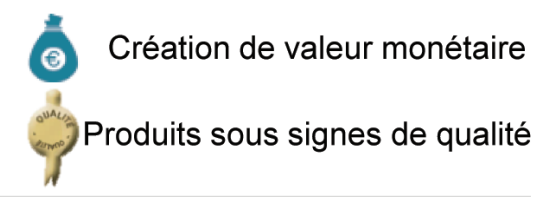

\section{Intrants}

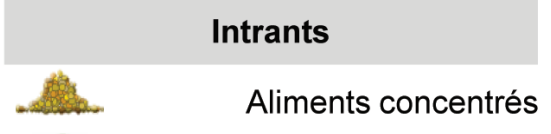

Engrais, pesticides, énergie

\section{Enjeux sociaux et culturels}

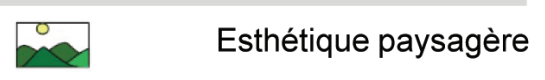

Patrimoine gastronomique

Aspects réglementaires et juridiques

Travail et emploi

Coordination entre acteurs 
Figure 3. Frise temporelle de l'AOP Laguiole.

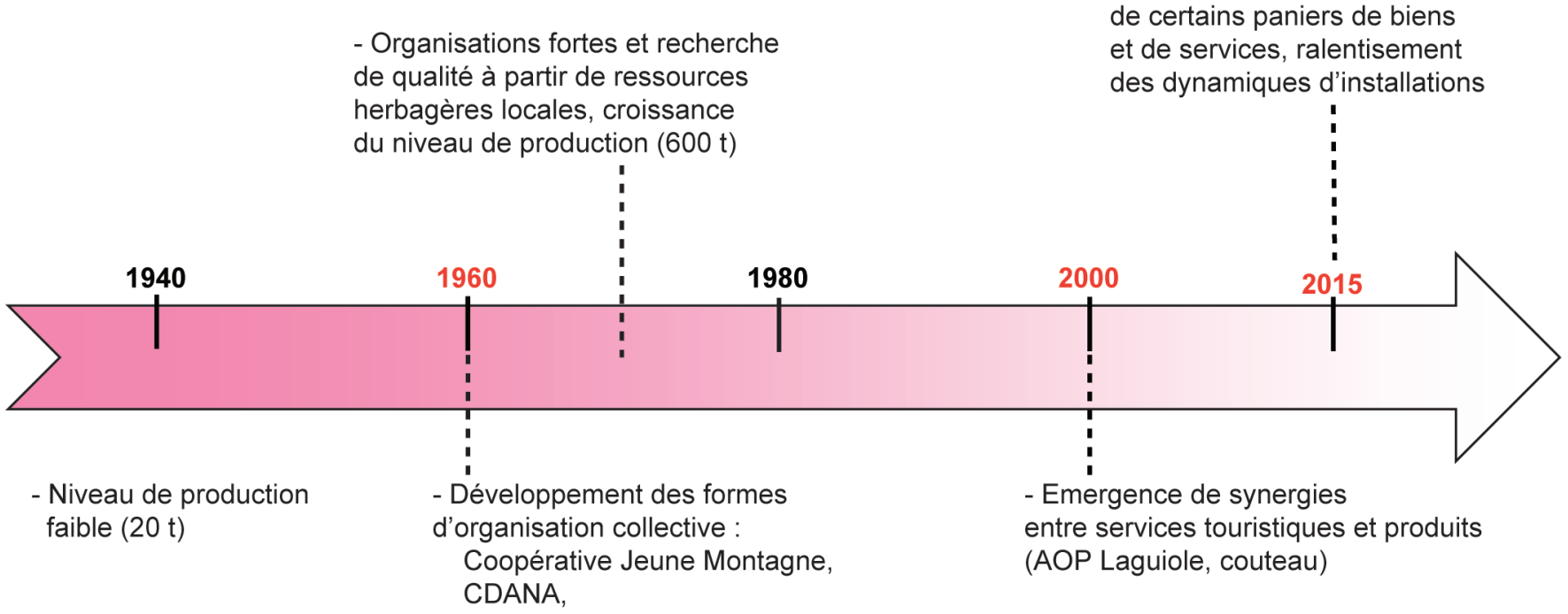

de la chambre d'agriculture, qui s'est même décentralisé au niveau local (le CDANA). La coopérative d'élevage du Massif central a été créée en 1966, à Laguiole sur ces mêmes bases (figure 3). Ces formes d'organisation collective se sont révélées efficaces pour capter de la valeur ajoutée. La recherche de qualité se traduit par la mise en place de nouveaux signes de qualité (CCP Fleur d'Aubrac, Label Rouge Bœuf Fermier Aubrac, projet IGP Aligot d'Aubrac) ou de signes de qualité supplémentaires pour ceux déjà existants (suppression de l'ensilage). La coopérative Jeune Montagne a conduit à abandonner la fabrication individuelle des buronniers (mise en place par les grands propriétaires fonciers regroupés depuis 1798 au sein de la société centrale d'agriculture de l'Aveyron) au profit d'un fonctionnement plus solidaire. Les exigences du cahier des charges se sont progressivement renforcées avec les décrets de 1986 et 2000 (avec notamment la suppression de l'ensilage). L'aligot (mélange de tomme fraiche et de pomme de terre) participe aussi à la construction identitaire de la région. Un projet «IGP Aligot de l'Aubrac » a été porté par la coopérative Jeune Montagne jusqu'ici sans succès en raison de contraintes liées à la réglementation européenne.

\section{b) Des services d'approvisionnement générateurs d'emploi local}

Protégé par une AOC depuis 1961, le fromage Laguiole a retrouvé en 1980 son niveau de 1920 (c'est-à-dire 700 tonnes contre 20 après-guerre). De surcroît, les innovations et les activités de promotion autour de l'aligot (aligot frais et surgelé en barquette, en kit) ont multiplié par six la production d'aligot et de tome depuis le milieu des années 80 ; l'aligot représente un peu moins de la moitié du chiffre d'affaires de la coopérative. L'emploi direct et indirect généré localement est très significatif : les 15 millions de litres récoltés annuellement auprès des 130 adhérents sont transformés localement par les 90 salariés de la coopérative. Il existe aussi quelques producteurs fermiers qui renforcent l'image de marque du produit.

c) Des formes de vitalités territoriales prometteuses mais limitées au territoire de l'Aubrac: des paniers de biens et de services

Contrairement à d'autres territoires du Massif central, l'Aubrac (qui est au cœur de la zone de production de l'AOP Laguiole) parvient à susciter de véritables complémentarités entre services et à créer une rente de qualité territoriale pour les produits locaux. On parle alors de « panier de biens et de services », c'est-à-dire des biens et services de qualité complémentaires notamment en termes de commercialisation (encadré 1).

Le panier de biens et de services de l'Aubrac inclut non seulement une offre spécifique de produits (fromage AOP, couteau, aligot), mais aussi des services touristiques complémentaires à partir des gîtes ruraux, comme des circuits de visite et de la restauration (Mollard et al 2006, Vollet 2014). Cette offre rencontre une demande qui se caractérise par un fort attrait pour des produits de qualité misant sur leur lien au territoire.

Une partie des facteurs favorables à la consolidation de ce processus est fragile. Il existe certes une bonne coordination des acteurs privés pour les filières du fromage, mais celle-ci est en cours de consolidation pour le tourisme et la viande bovine grâce à quelques initiatives originales (valorisation des jeunes femelles de 18 mois en race pure Aubrac ou croisée Aubrac-Charolais). Les coordinations sont à construire pour la filière coutelière (Angeon et Vollet 2008). Parallèlement, des coordinations institutionnelles se développent autour d'un projet de Parc Naturel Régional avec des acteurs publics et privés (Mollard 2014). La pression foncière très forte sur l'Aubrac ralentit cependant les installations (Laurent 2014).

\section{Encadré 1. Définition du panier de biens et de services.}

Un panier de biens et de services se caractérise par la rencontre entre l'offre et la demande pour des produits de qualité.

L'offre ancrée dans un territoire donné confère à ces produits un caractère exclusif (non accessible ailleurs), interdépendant et complémentaire. Ce caractère résulte d'une « spécificité » qui n'est pas seulement donnée, comme une dotation initiale de ressources ou de facteurs localisés, mais qui est aussi construite par les acteurs du territoire.

La demande conjointe de produits et services de qualité dépend certes du prix et de signaux officiels et objectifs, mais surtout d'éléments à caractère subjectif et affectif, donc d'attributs cognitifs non codifiés, d'images ou de symboles. 
Les principaux impacts de l'AOP Laguiole représentés de manière synthétique dans la figure 4 et le tableau 2 sont les suivants :

i) Une production significative de produits de qualité (comparable à son niveau maximum du début du vingtième siècle mais très inférieure à celle du Cantal) qui s'appuie sur l'existence d'un cahier des charges exigeant et valorise les savoir-faire locaux et le patrimoine culturel ;

ii) un bon niveau de rémunération des emplois agricoles en raison d'une gouvernance territoriale ;

iii) une production notable de services environnementaux (biodiversité notamment) qui s'appuie sur la diversité des prairies permanentes et bénéficie de l'interdiction totale de l'ensilage.
Figure 4. Synthèse des services et impacts rendus par le système AOP Laguiole.

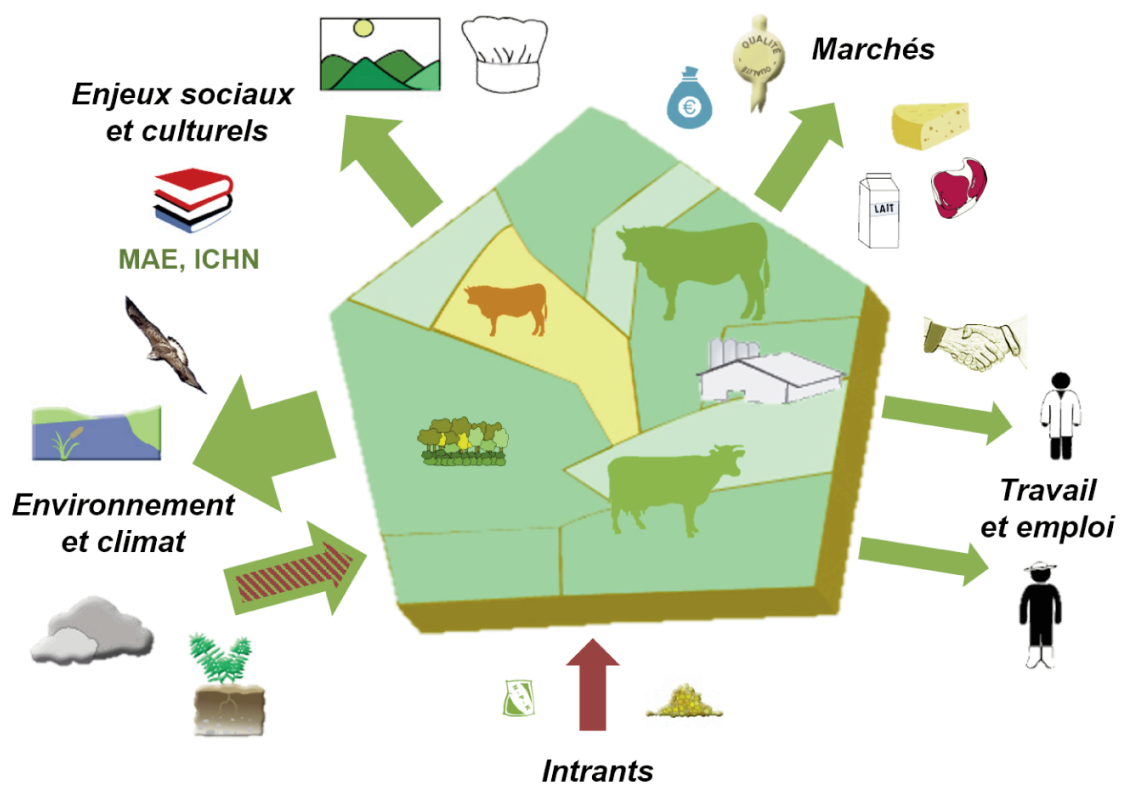

Tableau 2. Effets positifs et négatifs associés aux systèmes d'élevage de l'AOP Laguiole.

\begin{tabular}{|c|c|c|}
\hline Interfaces étudiées & Effets positifs & Effets négatifs \\
\hline Intrants & - Autonomie fourragère élevée & $\begin{array}{l}\text { - Effets délocalisés assez limités de la } \\
\text { production d'aliments concentrés }\end{array}$ \\
\hline Travail et emploi & $\begin{array}{l}\text { - Nombre significatif d'emplois directs bien } \\
\text { rémunérés } \\
\text { - Emploi indirect dans une filière bien } \\
\text { organisée pour capter valeur ajoutée }\end{array}$ & \\
\hline Marchés & $\begin{array}{l}\text { - Nombreux signes de qualité } \\
\text { - Volumes limités qui concourent à une } \\
\text { valorisation économique élevée }\end{array}$ & - Saisonnalité de la production à l'herbe \\
\hline $\begin{array}{l}\text { Effets sociaux } \\
\text { et culturels }\end{array}$ & $\begin{array}{l}\text { - Esthétique du paysage, identité culturelle } \\
\text { - Patrimoine gastronomique (aligot) } \\
\text { - Préservation de savoir-faire locaux } \\
\text { - Qualité sensorielle et nutritionnelle } \\
\text { des produits à l'herbe }\end{array}$ & \\
\hline $\begin{array}{l}\text { Environnement } \\
\text { et climat }\end{array}$ & $\begin{array}{l}\text { - Biodiversité (prairies permanentes, } \\
\text { interdiction totale de l'ensilage, habitats } \\
\text { remarquables, race Aubrac) } \\
\text { - Séquestration du carbone } \\
\text { - Qualité des eaux }\end{array}$ & $\begin{array}{l}\text { - Émissions élevées de } \mathrm{CH}_{4} \text { par kg de produit } \\
\text { - Sensibilité aux aléas climatiques et aux } \\
\text { ravageurs (campagnols terrestres ou rats } \\
\text { taupiers) }\end{array}$ \\
\hline
\end{tabular}

\section{3 / Franche-Comté}

\section{1 / Un contexte et un système productif très spécifique lié à la filière Comté}

Le contexte de la Franche-Comté est très marqué par celui d'une filière fromagère, celle du Comté, dont le système productif reste original. Quatre étapes peuvent être identifiées dans la constitution de la filière Comté (Perrier-Cornet
1990, Jeanneaux et Perrier-Cornet 2011) (figure 5). Une première phase a visé à reconnaître l'exclusivité d'un droit à produire territorialisé. À ce titre, le comté bénéficie de l'AOC depuis 1952. La deuxième étape a poursuivi cette dynamique et a conduit à des normes de production permettant une différenciation marquée du produit. Les décrets de 1976, 1979, 1986, 1994, y ont largement contribué. Dans une troisième phase, les producteurs ont cherché à maîtriser l'offre fromagère. Différents facteurs ont conduit au retrait des zones basses de la zone de production. Désormais, la régulation de la production régionale est organisée autour de plans de campagne successifs assortis d'une clause de sauvegarde. Ces mesures semblent éviter les crises de productions auxquelles les professionnels pourraient être confrontés. Depuis les années 2000, des facteurs externes perturbent la stratégie du système producteur fromager franccomtois, en particulier l'arrivée de grands groupes nationaux, et la modification des conditions techniques d'affinage. 
Figure 5. Frise temporelle de l'AOP Comté.

- Elaboration de normes de production nécessaires à la différentiation du produit (décrets de 1976, 1979, 1986) : fourrages secs uniquement, délai d'emprésurage limité à $24 \mathrm{~h}$, durée minimale d'affinage

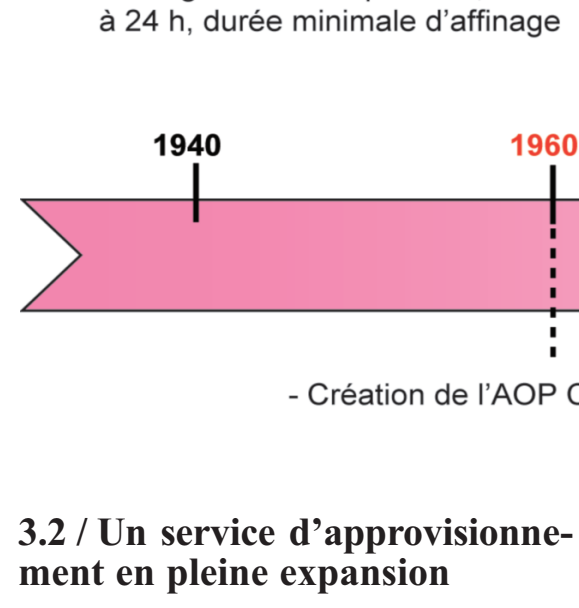

Le massif jurassien de Franche-Comté se caractérise par la production de fromages sous signe de qualité reposant sur la relation entre coopératives-fruitières et négociants-affineurs. Il a notamment permis aux éleveurs d'obtenir des prix du lait très supérieurs au prix national depuis une trentaine d'années. Cette réussite s'explique par la création d'une rente d'appellation ${ }^{2}$ et par la capacité des éleveurs du massif rassemblés dans leurs fruitières à en capter une partie.

Les acteurs traditionnels de la filière ont réussi à faire adopter en 1976 un décret défendant la spécificité d'un modèle de production à l'origine de la rente d'appellation. Ce décret instituait le modèle de production fromager « montbéliard, coopératif et jurassien 》 (PerrierCornet 1990) sur lequel se fonde le modèle actuel de production en filière de l'AOC Comté. Il contient des obligations, qui s'appliquent aux trois principaux maillons de la filière :

i) Les éleveurs producteurs de lait ne peuvent produire du lait qu'avec des vaches de races locales (Montbéliarde et Pie rouge de l'Est), dont l'alimentation comprend exclusivement des fourrages secs et non des fourrages fermentés ;

ii) dans les ateliers des coopérativesfruitières de première transformation, le délai d'emprésurage du lait est limité à $24 \mathrm{~h}$ maximum après la première traite (36 $\mathrm{h}$ en hiver), et la thermisation du lait est interdite ;

iii) les affineurs-négociants qui mettent en marché doivent depuis 1994 respecter
- Des signes de fragilité du modèle traditionnel

- Des premiers effets sur la biodiversité prairiale 
Gillet et al (2016) ont cependant montré que les prairies de Franche-Comté avaient subi une diminution de leur diversité floristique au cours des vingt dernières années en raison de l'intensification des pratiques agricoles, et ceci malgré un cahier des charges exigeant vis-à-vis du chargement et de la fertilisation minérale (Mauchamp 2014). Pour remédier aux aléas climatiques, certains agriculteurs adoptent des pratiques de fertilisation plus intensives et multiplient les coupes de fourrages pour maximiser les stocks hivernaux. La production laitière est accrue, mais en augmentant la part des concentrés produits hors du territoire. L'augmentation de la production laitière par vache fait que les Montbéliardes à haut potentiel génétique s'accommodent de moins en moins de fourrages grossiers. L'intensification de la conduite des prairies, ainsi que la part croissante de prairies temporaires réduisent la biodiversité prairiale (Michaud et Jeanneaux 2014). Des mesures complémentaires sur l'avifaune (Michelat et al 2015) concluent que des mesures environnementales supplémentaires seraient nécessaires au maintien de la biodiversité régionale.

\section{4 / Une vitalité territoriale cer-} taine qui s'appuie sur une bonne rémunération du lait

La filière Comté permet de faire vivre un réseau dense de producteurs. Environ 3000 éleveurs sont regroupés en petites coopératives de transformation du lait (130 fruitières en 2013) qui contrôlent
Figure 6. Synthèse des impacts et services rendus par le système AOP Comté.

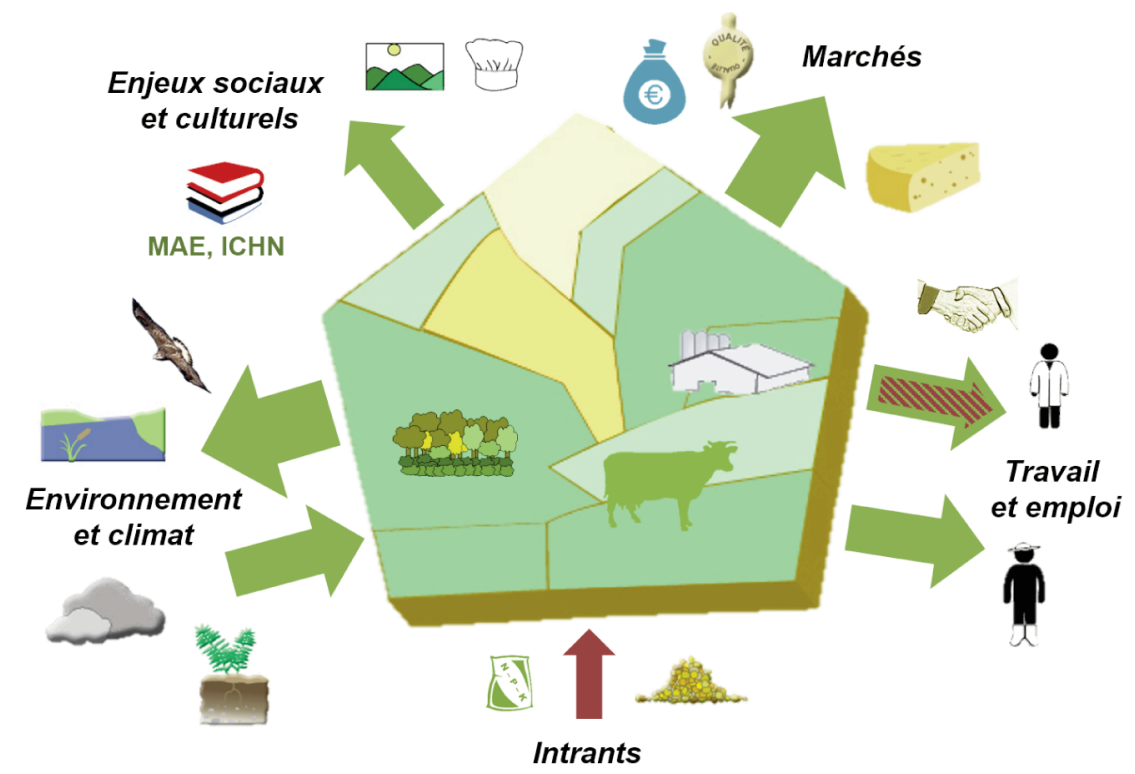

la production de fromage en blanc (préaffiné) mais n'ont pas accès au marché final. Les entreprises d'affinage (une dizaine en 2013) dominent l'accès au marché, mais n'investissent pas la première transformation. Une interprofession puissante régit le système. Le Comité interprofessionnel du comté (CIGC) contrôle l'offre des volumes de fromage et entretient un rapport de force équilibré entre éleveurs et affineurs par la moralisation de la transaction marchande du fromage en blanc. Celle-ci a été instaurée dans les années 1970 , via des règles précises de détermination du prix du fromage en blanc en fonction du cours du marché final. Cette forme de coopération produit un surplus collectif protégé par le cadre réglementaire de l'AOP. Elle a permis aux producteurs laitiers d'avoir un prix du lait durablement supérieur à la moyenne nationale durant les trente dernières années (par exemple, $+15 \%$ en 2010 : Diallo 2017).

En résumé, les principaux impacts positifs ou négatifs du système productif régional franc-comtois représentés de manière synthétique dans la figure 6 et le tableau 3 sont les suivants :

i) Une production importante et croissante de fromages AOP grâce à des moda-

Tableau 3. Effets positifs et négatifs associés aux systèmes d'élevage de l'AOP Comté.

\begin{tabular}{|c|c|c|}
\hline Interfaces étudiées & Effets positifs & Effets négatifs \\
\hline Intrants & - Autonomie fourragère élevée & $\begin{array}{l}\text { - Effets délocalisés production de concentrés } \\
\text { protéiques } \\
\text { - Fertilisation des prairies }\end{array}$ \\
\hline Travail et emploi & $\begin{array}{l}\text { - Nombreux emplois directs et indirects } \\
\text { (transformation) } \\
\text { - Rémunération élevée de tous les maillons de } \\
\text { la filière du fait de son organisation }\end{array}$ & $\begin{array}{l}\text { - Menaces liées à la prise de contrôle de la } \\
\text { filière par des grands groupes } \\
\text { - Changements technologiques d'affinage }\end{array}$ \\
\hline Marchés & $\begin{array}{l}\text { - Production massive de produits } \\
\text { de qualité } \\
\text { - Valorisation économique élevée (cahiers des } \\
\text { charges stricts, innovation, réputation) }\end{array}$ & \\
\hline $\begin{array}{l}\text { Effets sociaux } \\
\text { et culturels }\end{array}$ & $\begin{array}{l}\text { - Esthétique du paysage, identité culturelle } \\
\text { - Patrimoine gastronomique } \\
\text { - Qualité sensorielle et nutritionnelle des } \\
\text { produits à l'herbe }\end{array}$ & \\
\hline $\begin{array}{l}\text { Environnement } \\
\text { et climat }\end{array}$ & $\begin{array}{l}\text { - Biodiversité (prairies permanentes, habitats } \\
\text { remarquables } \\
\text { - Séquestration du carbone } \\
\text { - Qualité des eaux } \\
\text { - Valorisation services intrants }\end{array}$ & $\begin{array}{l}\text { - Émissions élevées de } \mathrm{CH}_{4} \text { par kg de produit } \\
\text { - Sensibilité aux ravageurs (rats taupiers) } \\
\text { - Risques liés à l'intensification }\end{array}$ \\
\hline
\end{tabular}


lités particulières de coopération entre éleveurs-fruitières et affineurs reposant sur l'existence de cahiers des charges stricts et en recherche continue de progression : le système de production se caractérise par un modèle de production et de valorisation du lait et du fromage lié à une capacité d'innovation sectorielle territorialisée et à la réputation du fromage (Dervillé 2012) ;

ii) une rémunération élevée de tous les maillons de la filière en raison de sa capacité d'organisation ;

iii) une production notable de services environnementaux (biodiversité, qualité de l'eau).

\section{4 / Estives des Alpes suisses}

\section{1 / Un contexte marqué par une arrivée récente de la labélisation AOP}

La frise temporelle de cette région (figure 7) souligne l'importance de la politique agricole sur la dynamique de production laitière. L'appellation « alpage » (fromage d'alpage AOP) est nettement plus tardive que pour les AOP du Massif central présentées précédemment et la Franche-Comté. Une évolution différenciée des parcelles d'estives est observée de longue date. Tandis que les parcelles les plus accessibles et les plus fertiles sont intensifiées, les autres s'enfrichent progressivement. Depuis 2014, la possibilité d'obtenir des primes (contributions) de qualité de la biodiversité et de qualité du paysage pour les exploitations d'estivage pourrait maintenir une utilisation agricole des parcelles difficilement accessibles.

\section{2 / Un service d'approvisionne- ment significatif et en lien fort avec l'identité locale}

En Suisse les estives représentent une surface additionnelle importante pour la production de fourrage des exploitations de plaine ou de basse montagne (Fischer et al 2012), et ceci bien que la productivité des parcelles y soit nettement plus faible. Ces alpages (Jura compris) couvrent une superficie qui équivaut à presque la moitié de la surface agricole utile (Office fédéral de la statistique 2015). Le fourrage produit annuellement sur les alpages correspond à l'équivalent de fourrage grossier nécessaire à 80000 UGB pendant une année (Mack et Flury 2014). Une partie du lait produit sur les estives est valorisée sous forme de fromage d'alpage, à savoir de fromage fabriqué sur l'estive durant l'été. Avec 5230 tonnes de fromage, dont environ la moitié est commercialisée sous AOP, ils représentent $2,9 \%$ de la production fromagère suisse (Herzog et al 2014). Selon Böni et al (2014), la production de fromage d'alpage se vend bien et le consommateur suisse est prêt à payer un prix 8 à $30 \%$ plus élevé pour un fromage d'alpage AOP que pour un fromage de plaine. Les volumes produits ont ainsi augmenté de plus de $15 \%$ entre 2003 et 2009 (Böni et Seidl 2012).

La vente directe et les magasins de village représentent leur principal canal de distribution (Böni and Seidl 2012), ce qui montre le fort lien qui existe entre la filière agricole et la filière touristique dans ces territoires. La motivation des consommateurs pour ces produits différerait entre la Suisse alémanique où les raisons liées à la qualité primeraient, et les régions francophones où les motivations liées à la régionalité des produits seraient prioritaires (Böni et Seidl 2012). Les spécificités des laits et des fromages à pâte pressée cuite produits en alpage ont été décrites par différents auteurs (Bosset et al 1999, Bugaud et al 2002). La matière grasse du lait et des fromages produit sur l'alpage contient une proportion plus importante d'acides gras insaturés que celle du lait produit en plaine (Collomb et al 2002) ce qui est considéré comme positif vis-à-vis de la qualité nutritionnelle des produits laitiers. Les fromages d'alpage ont également une flaveur plus corsée (Bosset et al 1999).

La production agricole sur les estives est à la fois fortement influencée par les contributions d'estivage (Mack et Flury 2014), ainsi que par une législation fixant la charge en bétail et limitant fortement les apports d'aliments concentrés. D'après une étude ayant analysé 18 alpages suisses, deux tiers du revenu dégagé par les alpages proviendrait de la vente des produit et un tiers des subventions (Blättler et al 2013).

\section{3 / Une qualité environnemen- tale avérée de par la biodiversité prairiale}

Les pelouses des estives sont un hotspot de biodiversité en Europe (Fischer et al 2012, Riedel et al 2012). Ainsi, les trois quarts des surfaces de pâturages secs et de bas-marais d'importance nationale se trouvent dans la région d'estivage (Herzog et al 2014). Les enjeux relatifs

Figure 7. Frise temporelle de l'élevage dans les estives des Alpes suisses.

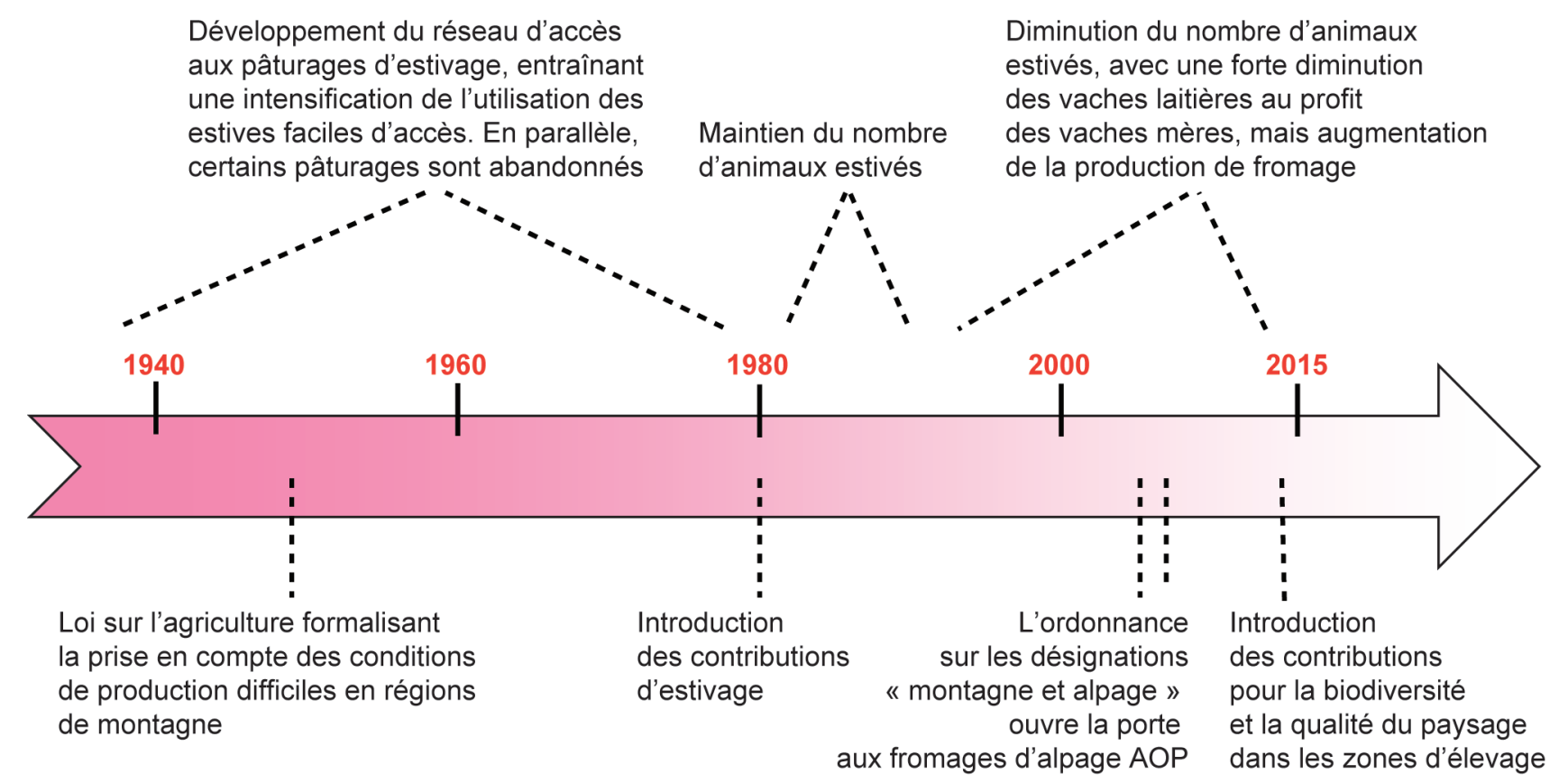


à la biodiversité sont donc majeurs dans ces territoires. Peter et al $(2008,2009)$ ont montré que la diversité végétale des prairies de l'arc alpin s'était en général maintenue durant ces dernières décennies, aussi bien dans les prairies non fertilisées que dans les fertilisées. Néanmoins, l'augmentation de la fertilisation pourrait à terme entraîner une diminution de la richesse floristique des couverts (Homburger et Hofer 2012). Dans les prairies de montagnes, une fertilisation modérée est avantageuse pour la qualité du fourrage produit (Tenz et al 2010) alors qu'une fertilisation élevée sélectionne des dicotylédones compétitives de valeur agronomique médiocre (Pauthenet et al 1994). On observe une tendance récurrente à une polarisation de la gestion des prairies (Tasser et al 2007), avec une intensification des surfaces faciles d'accès et un abandon des surfaces marginales (Gellrich et al 2007). Celle-ci représente une menace pour la biodiversité de ces habitats, conséquence des processus présentés au paragraphe 1.2.

Le loup est présent en faible nombre dans les Alpes suisses ${ }^{4}$, mais provoque déjà d'importants conflits entre éleveurs et protecteurs de la nature. Il scinde les opinions sur les possibilités de maintenir en sa présence la fourniture d'un service d'approvisionnement et d'entretien du paysage (Gigon 2015). Ces conflits souvent médiatisés altèrent la perception du rôle de l'élevage pour la préservation de la biodiversité dans les régions concernées. En comparant le nombre d'animaux de rente dédommagés en Suisse suite à une attaque avérée de loup aux pertes totales de moutons durant la période d'estivage (Werder 2012), leur prédation ne représenterait qu'environ $6 \%$ des pertes de petits ruminants au pâturage (Seidl et al 2014). Les troupeaux non protégés peuvent cependant subir de lourdes pertes (Willisch 2014). Le loup pose donc déjà un réel défi à quelques éleveurs et pourrait à terme entraîner l'abandon de certaines estives (Seidl et al 2014). Ceci n'a cependant pas encore été observé dans la région de Gantrisch-Schwarzsee où la présence du loup est attestée depuis 2009 (Willisch 2014).

\section{4 / Une vitalité territoriale sans lien fort avec l'agrotourisme et le travail agricole}

L'office fédéral de l'agriculture indique que « les contributions d'estivage ont pour objectif d'assurer l'exploitation et l'entretien des vastes pâturages d'estivage dans les Alpes, les Préalpes et le Jura » (OFAG 2014); l'occupation du territoire

Figure 8. Synthèse des impacts et services rendus par l'élevage dans les alpages suisses.

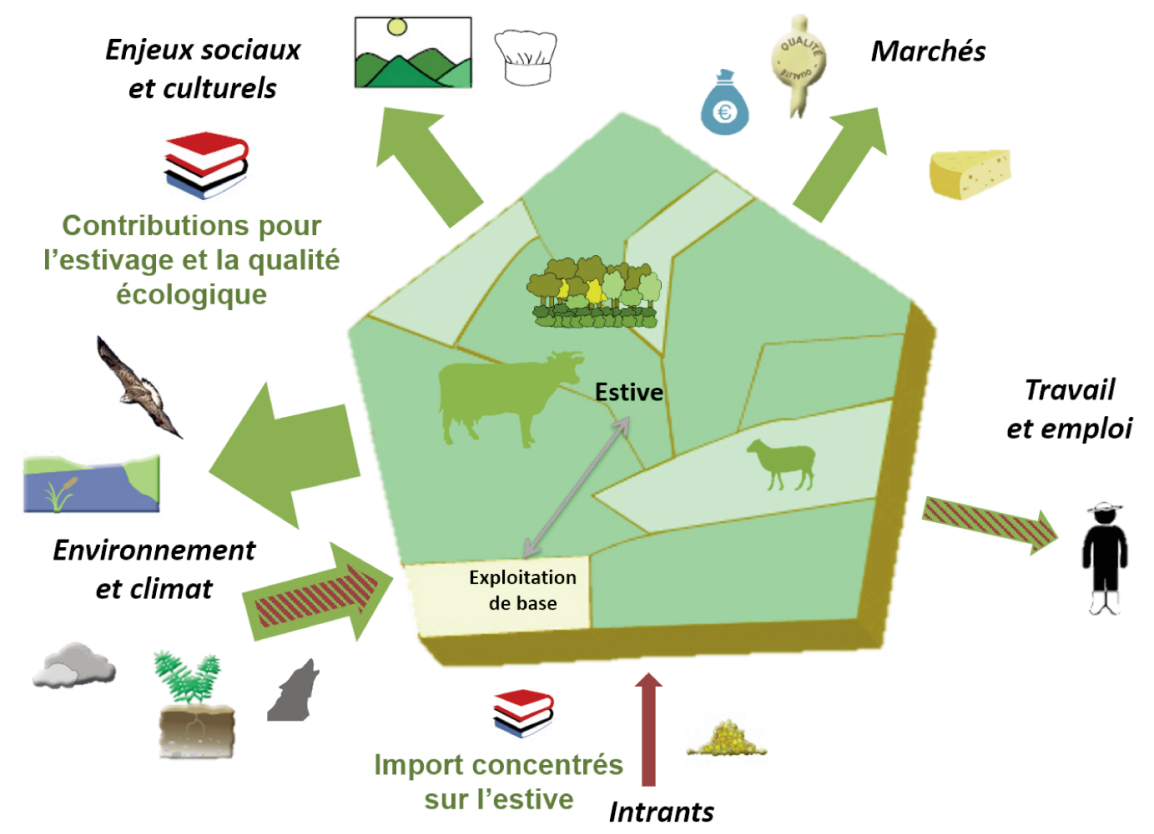

apparait ainsi comme un objectif majeur de l'activité agricole sur les estives Suisses. Les services écosystémiques de conservation de l'héritage culturel ainsi que ceux en rapport au tourisme sont majeurs dans les pâturages alpestres (Bürgi et al 2015) qui contribuent à l'identité nationale (Junge et Hunziker 2013). Cependant, malgré les nombreux sentiers pédestres et les autres infrastructures touristiques, l'agrotourisme n'est que peu développé sur les alpages suisses en dehors de la restauration proposée par les buvettes d'alpage (Seidl et al 2014). Un certain taux de reboisement de ces paysages ne semble pas affecter la perception de leur esthétique scénique (Seidl et al 2014). La plupart des pistes de ski utilisent néanmoins les pâturages. Le travail sur les exploitations d'alpage ne représente qu'une petite part du marché du travail des régions concernées qui culmine à 17000 personnes durant la période d'estivage (Mann et Calabrese 2014). C'est avant tout le style de vie qui motive la décision de ceux qui travaillent en alpage malgré un travail dur et une rémunération modeste (Calabrese et al 2014). Même si l'importance de l'utilisation agricole des estives est incontestable pour la vitalité des territoires de montagne, son impact sur l'économie dans les Alpes suisses n'a, à notre connaissance, pas été quantifié.

En résumé, les principaux impacts et services rendus par l'élevage dans les alpages suisses sont représentés de manière synthétique dans la figure 8 et le tableau 4 : i) Une production significative et très identitaire de fromages AOP depuis la promulgation dans les années 2000 de l'ordonnance sur les désignations alpage ;

ii) une rémunération variable du travail agricole (et particulièrement faible pour les salariés) ;

iii) une production remarquable de services environnementaux liée aux conditions contenues dans les contributions pour l'estivage et la qualité écologique, notamment en termes de biodiversité prairiale. Celle-ci pourrait toutefois être à terme menacée par de mauvaises pratiques de gestion et par la présence du loup.

\section{5 / Quels leviers d'action pour les territoires herbagers de montagne?}

L'analyse des bouquets de services que nous avons conduite dans quatre territoires met en évidence différents leviers pour concilier les performances productives, environnementales et sociales des systèmes herbagers (tableau 5). Une préoccupation majeure consiste à accroître l'autonomie fourragère des exploitations, avec une logique d'adaptation des pratiques aux potentialités du milieu (Dumont et al 2017). Ainsi, dans les zones fromagères AOP, les innovations cherchentelles à optimiser la gestion du système fourrager, voire à supprimer l'usage des concentrés dans des systèmes plus exten- 
Tableau 4. Effets positifs et négatifs associés à l'élevage dans les alpages suisses.

\begin{tabular}{|c|c|c|}
\hline Interfaces étudiées & Effets positifs & Effets négatifs \\
\hline Intrants & $\begin{array}{l}\text { - Réglementation sur niveaux de concentrés } \\
\text { importés } \\
\text { - Autonomie fourragère sans fertilisation } \\
\text { minérale }\end{array}$ & $\begin{array}{l}\text { - Effets délocalisés liés aux concentrés très } \\
\text { limités }\end{array}$ \\
\hline Travail et emploi & $\begin{array}{l}\text { - Emploi agricole quantitativement limité } \\
\text { - Satisfaction par rapport au mode de vie }\end{array}$ & - Faible rémunération des salariés agricoles \\
\hline Marchés & $\begin{array}{l}\text { - Produits SIQO reconnus (désignation alpage) } \\
\text { - Valorisation économique élevée }\end{array}$ & \\
\hline $\begin{array}{l}\text { Effets sociaux } \\
\text { et culturels }\end{array}$ & $\begin{array}{l}\text { - Esthétique du paysage } \\
\text { - Identité culturelle } \\
\text { - Préservation de savoir-faire locaux } \\
\text { - Qualité sensorielle et nutritionnelle des } \\
\text { produits }\end{array}$ & \\
\hline $\begin{array}{l}\text { Environnement } \\
\text { et climat }\end{array}$ & $\begin{array}{l}\text { - Biodiversité (prairies d'alpage, habitats } \\
\text { remarquables, races : Grise rhétique, Hérens) } \\
\text { - Séquestration du C } \\
\text { - Qualité des eaux }\end{array}$ & $\begin{array}{l}\text { - Conflits liés à la présence de grands } \\
\text { prédateurs } \\
\text { - Emissions de } \mathrm{CH}_{4} \text { élevées par kg de } \\
\text { produit }\end{array}$ \\
\hline
\end{tabular}

Tableau 5. Principaux leviers d'action permettant de concilier performances productives, environnementales et sociales dans les territoires herbagers.

\begin{tabular}{|c|c|}
\hline $\begin{array}{l}\text { Échelle } \\
\text { Troupeau } \\
\text { Exploitation }\end{array}$ & $\begin{array}{l}\text { Raisonner l'utilisation des prairies par une approche systémique de l'alimentation, de la } \\
\text { santé et de l'environnement } \\
\text { Raisonner la conduite du pâturage en fonction de compromis entre production et } \\
\text { environnement : } \\
\text { - Utilisation différenciée des parcelles en fonction de leur potentiel de production (conditions } \\
\text { pédoclimatiques et topographiques) et de leur potentiel pour la conservation de la biodiversité } \\
\text { - Favoriser le pâturage ou la fauche tardive, exclure temporairement certaines parcelles } \\
\text { du pâturage (surfaces de promotion de la biodiversité en Suisse...) } \\
\text { - Conserver des prairies riches en légumineuses et des infrastructures agro écologiques } \\
\text { présentes sur l'exploitation (haies, bandes enherbées...) } \\
\text { Repenser la gestion du troupeau en lien avec la pousse de l'herbe : } \\
\text { - Mettre en cohérence les besoins alimentaires des troupeaux (période de vêlages, types } \\
\text { d'animaux...) et la qualité des fourrages (conduite des prairies...) } \\
\text { - Réduire l'usage des aliments concentrés dans des systèmes extensifs valorisant } \\
\text { une diversité de prairies permanentes } \\
\text { - Développer la mixité des troupeaux (ovins, bovins, équins) pour valoriser plus complètement } \\
\text { l'herbe et limiter le parasitisme par effet de dilution }\end{array}$ \\
\hline $\begin{array}{l}\text { Échelle } \\
\text { Territoire }\end{array}$ & $\begin{array}{l}\text { Organisation territoriale pour des projets à haute valeur environnementale et } \\
\text { économique } \\
\text { Favoriser les organisations paysagères complexes (diversité de types de prairies, } \\
\text { connectivité de la trame verte à travers les haies, les arbres isolés, les murets...) } \\
\text { - Développer de nouvelles formes d'organisations collectives pour la mise en réseau } \\
\text { des habitats pour la flore et la faune, pour la valorisation des haies en bois énergie, etc. } \\
\text { Développer des cercles d'information et lieux d'apprentissages : } \\
\text { - Faire échanger les agriculteurs sur l'effet de leurs pratiques sur les services écosystémiques } \\
\text { - Développer des discussions collectives entre acteurs locaux concernés par les évolutions de } \\
\text { l'élevage (éleveurs et acteurs de la filière, tourisme, ONG...) } \\
\text { Maintenir et développer des filières de produits sous Signes d'Identification de la Qualité } \\
\text { et de l'Origine (SIQO) : } \\
\text { - Raisonner les gouvernances territoriales des filières de qualité caractérisées par la } \\
\text { transparence des prix et des mécanismes institutionnels de régulation (AOP Cantal) } \\
\text { - Développer la communication dans le domaine de la typicité et de la qualité environnementale } \\
\text { des fromages }\end{array}$ \\
\hline
\end{tabular}


sifs (Farruggia et al 2014). La diversité des parcelles d'une exploitation offre des leviers pour adapter leur conduite aux conditions pédoclimatiques et conduit à des synergies entre la valeur agronomique des prairies, leur souplesse d'utilisation et la conservation de la biodiversité. Un verrou qui limite l'adoption des fauches tardives, positives pour la biodiversité, est le besoin d'un fourrage riche en énergie et en protéines pour nourrir les animaux. Optimiser le fonctionnement du système fourrager permet de disposer de fourrages de bonne valeur nutritive plus longtemps dans la saison. Le pic de production laitière est moins élevé mais la production plus stable, pour des niveaux de production finalement équivalents à l'échelle d'une lactation (Farruggia et al 2014). Les laits à base d'herbe ont un profil en acides gras intéressant. La maîtrise de la reproduction dans les systèmes laitiers bas intrants reste un verrou majeur (Pires et al 2015).

Le Casdar ATOUS a permis de cartographier la diversité des types de prairies et des services fourragers et écosystémiques à l'échelle de petits territoires du Massif central et des Alpes du Nord (Carrère et al 2015). Les discussions autour de ces cartes constituent un lieu d'échange et d'apprentissage pour les éleveurs, qui leur fait prendre conscience des effets de leurs pratiques sur les services écosystémiques. Dans les prairies alpines, une démarche participative analogue a conduit à proposer différents scénarios de gestion collective d'un territoire. Audelà de la hiérarchisation des services, l'enjeu était de trouver des solutions d'aménagement de l'espace susceptibles d'être adoptées par l'ensemble des acteurs locaux (Lamarque et al 2014). Dans le Massif central et en Franche-Comté, préserver les haies pourrait aussi limiter les pullulations de rats taupiers, en fai- sant obstacle au développement de leurs galeries et en offrant des abris à leurs prédateurs (Note et Michelin 2014).

Plus largement, la gouvernance joue un rôle tout particulier dans ce type d'espace (tableau 6) et cela indépendamment des volumes produits qui peuvent être très variables pour un mode de gouvernance donné. L'efficacité des organisations locales conditionne la mise en place de cahiers des charges suffisamment restrictifs (du point de vue de la délimitation, des conditions de production, des divers types de qualité) pour être reconnus par les consommateurs, tout en étant partagés par les producteurs. Celle-ci se traduit au niveau du prix payé au producteur pour des services environnementaux rendus par les systèmes de production, au travers de la conception des cahiers des charges. Une série de travaux menés depuis une trentaine d'années illustre ces liens (PerrierCornet 1990, Ricard 1994, 2013, Barjolle et al 2007, Diallo 2017). En ce qui concerne la valeur ajoutée, Barjolle et al (2007) ont montré que le prix payé au producteur pour les AOP Comté et Gruyère suisse a été de 15 à $25 \%$ supérieur à celui du lait générique, et ce sur une période longue. Inversement, aucune différence n'a été mise en évidence pour l'AOP Cantal caractérisée par une gouvernance sectorielle. Diallo (2017) confirme ces résultats à partir d'une analyse des résultats 2010 du RICA par une autre méthode économétrique (analyse des effets propres) : les filières AOP de la région Franche-Comté parviennent à générer des différences de valeur ajoutée significative, ce qui n'est pas le cas des AOP de la région Auvergne, dont l'AOP Cantal.

En raison du rôle important joué par la gouvernance, les leviers d'action poten- tiellement actionnables sont étroitement dépendants des facteurs en jeu dans chacun des types de gouvernance, surtout dans un contexte de passage d'une régulation publique (les quotas laitiers) à une régulation privée qui donne un rôle accru aux organisations de producteurs (You 2015). Les économies d'échelle constituent le principal facteur de compétitivité des produits dépendant d'une gouvernance sectorielle. Dans le cas d'une gouvernance territoriale, il s'agit plutôt d'économies de gamme : les services rendus par l'élevage peuvent alors être plus variés. D'autres auteurs ont décrit une forme de gouvernance très spécifique non rencontrée dans nos études de cas (du moins à ce stade de leur évolution) : la gouvernance alimentaire territoriale (Chiffoleau et Prévost, 2012). Les innovations et surtout des approches intégrées de l'innovation constituent alors des leviers privilégiés. Il peut s'agir d'innovations institutionnelles (comme les Projets Alimentaires Territoriaux ou les espaces tests agricoles) ou sociales (comme de nouvelles formes de propriété foncière telles que le portage foncier). Par ailleurs, il apparait de façon croissante des hybridations au sein même des territoires et à l'intérieur des filières entre ces types de gouvernance. Elles conduisent à des formes très originales de coexistence de projets voire de modèles de développement, souvent appuyées par les collectivités territoriales sur des thématiques partagées par plusieurs filières (voir l'exemple de la Picardie au sujet des contraintes d'organisation du travail dans les exploitations d'élevage dans l'encadré 3 de Ryschawy et al 2017, ce numéro).

Par exemple, dans le cas de l'AOP Laguiole, l'insertion de la filière dans une dynamique de Parc Naturel Régional pourrait à terme faire évoluer la gouver-

Tableau 6. Liens entre gouvernance des filières et services fournis.

\begin{tabular}{|l|c|c|c|c|}
\cline { 2 - 5 } \multicolumn{1}{c|}{} & \multicolumn{2}{c|}{ Massif central } & Franche-Comté & Estives suisses \\
\cline { 2 - 5 } \multicolumn{1}{c|}{} & AOP Cantal & AOP Laguiole & AOP Comté & Fromage d'alpage AOP \\
\hline Approvisionnement (en 2015) & $18000 \mathrm{t}$ & $670 \mathrm{t}$ & $52000 \mathrm{t}$ & $2500 \mathrm{t}$ \\
\hline Qualité environnementale & + & ++ & ++ & ++ \\
\hline Vitalité territoriale & + & ++ & ++ & ++ \\
\hline $\begin{array}{l}\text { Type de gouvernance } \\
\text { (Barjolle et Jeanneaux 2012) }\end{array}$ & Sectorielle $^{(\text {a) }}$ & Territoriale $^{(\mathrm{b})}$ & Territoriale $^{(\mathrm{b})}$ & Territoriale $^{(\mathrm{b})}$ \\
\hline
\end{tabular}

(a) Gouvernance sectorielle caractérisée par création de valeur par différenciation sur la technologie, distribution de la valeur par grille de prix national et absence de relation entre prix de vente du produit final et rémunération du lait, préservation de l'avantage compétitif par stratégie de coût et régulation du système de production par oligopole.

(b) Gouvernance territoriale caractérisée par création de valeur par valorisation de ressources locales spécifiques, distribution de la valeur par transparence des prix et mécanisme institutionnel, préservation de l'avantage compétitif par lien produit-terroir et régulation du système de production par organisations spécifiques. 
nance territoriale actuelle vers une forme de gouvernance alimentaire territoriale dans laquelle les innovations institutionnelles (visant à réguler le foncier et à renouveler les formes de relation avec les pôles urbains éloignés) auraient un rôle de levier majeur. Le " modèle de système productif local $\gg$ mis en application dans l'AOP Comté est quelque peu bousculé par l'arrivée de grands groupes nationaux qui modifient les équilibres entre acteurs et les procédés de transformation fromagère (Jeanneaux et Perrier-Cornet 2011). On peut se demander si ce serait le signe d'une hybridation plus forte entre gouvernances sectorielle et territoriale (ouvrant ainsi à la contractualisation un rôle plus fort que sur la période précédente). Inversement, l'AOP Cantal qui a renouvelé récemment son cahier des charges ne serait-elle pas en train d'évoluer vers une gouvernance certes essentiellement sectorielle mais mâtinée par les dimensions territoriales (développement de marchés de " niches » au sein de l'AOP, à l'image du « salers tradition salers pour l'AOP Salers) et alimentaires (par exemple, suite à la signature à titre expérimental d'un contrat de réciprocité ville-campagne entre le Grand Lyon et Aurillac)?

\section{Conclusion}

Les leviers d'action visant à favoriser la production des services produits par les territoires herbagers de montagne se situent à la fois au niveau de l'exploitation (par le raisonnement de la conduite du pâturage et de la gestion du troupeau) mais également du territoire via des formes de gouvernance plus ou moins favorables à l'émergence de mécanismes institutionnels de régulation. À l'échelle de l'exploitation, il s'agit de trouver un compromis entre une gestion extensive des prairies favorable à leur biodiversité et une gestion de l'herbe plus intensive qui accroît l'autonomie alimentaire des exploitations. Ces compromis doivent aussi prendre en compte le potentiel génétique des animaux et le bilan en éléments fertilisants à l'échelle du territoire. Au niveau des filières, une gouvernance territoriale plutôt que sectorielle semble plus propice à la fourniture d'un bouquet de services équilibré. Dans les exemples retenus en FrancheComté, dans l'Aubrac et les Alpes suisses les formes de gouvernances territoriales observées ont jusqu'ici permis de rémunérer les producteurs à des niveaux supérieurs à ceux des productions génériques. Elles ont maintenu voire développé la production et les emplois locaux, tout en fournissant nombre de services environnementaux. La gouvernance plus sectorielle de l'AOP Cantal se traduit par une moindre rémunération des producteurs, voire dans une moindre mesure par des services environnementaux dégradés selon les producteurs ou les territoires à l'intérieur de la zone d'appellation. Les formes d'hybridations qui émergent actuellement entre les formes de gouvernance sectorielle, territoriale et alimentaire pourraient à terme profondément renouveler les leviers d'action notamment ceux liés aux innovations sociales et institutionnelles.

\section{Références}

Andrieu N., Josien E., Duru M., 2007. Relationships between diversity of grassland vegetation, field characteristics and land use management practices assessed at the farm level. Agricult. Ecosys. Environ., 120, 359-369.

Angeon V., Vollet D., 2008. Spécificité des produits et développement territorial. L'exemple paradoxal du panier de biens en émergence de l'Aubrac. In : Revue d'Éonomie Régionale \& Urbaine, Colin A (Éd). 591-615.

Angers D.A., Arrouays D., Saby N.P.A., Walter C., 2011. Estimating and mapping the carbon saturation deficit of French agricultural topsoils. Soil Use Manage., 27, 448-452.

ANSES 2011. Actualisation des apports nutritionnels conseillés pour les acides gras. Rapport d'expertise collective, saisine 2006-SA-0359.

Barjolle D., Jeanneaux P., 2012. Raising rivals' costs strategy and localised agro-food systems in Europe. J. Food Syst. Dynam., 3, 11-21.

Barjolle D., Reviron S., Sylvander B., 2007. Création et distribution de valeur économique dans les filières de fromages AOP. Écon. Soc., 41, 1507-1524.

Bérodier F., 1997. Crus de Comté, flore des prairies et pratiques agricoles, Du terroir au goût des fromages. $5^{\text {th }}$ Plenary Meeting AIR 2039 - COST'95. Besançon, France, 186-189.

Blättler T., Durgiai B., Peguiron D., Raaflaub M., Winckler L., 2013. Efficience économique des alpages suisses. Rech. Agron. Suisse, 4, 108-115.

Böni R., Seidl I., 2012. Produits et services d'alpage - offre dans quelques régions ciblées de Suisse. Rech. Agron. Suisse, 3, 124-131.

Böni R., Seidl I., Junge X., 2014. Produits d'alpage : entre tradition et nouveautés. In: Avenir de l'économie alpestre suisse. Faits, analyses et pistes de réflexion du programme de recherche
AlpFUTUR. Lauber S., Herzog F., Seidl I., Böni R., Bürgi M., Gmür P., Hofer G., Mann S., Raaflaub M., Schick M., Schneider M., Wunderli R. (Éds). Birmensdorf, Institut Fédéral de Recherche WSL ; Zürich-Reckenholz, Station de recherche Agroscope, 94-109.

Bosset J.O., Jeangros B., Berger T., Butikofer U., Collomb M., Gauch R., Lavanchy P., Scehovic J., Troxler J., Sieber E.R., 1999. Comparaison de fromages à pâte dure de type gruyère produits en régions de montagne et de plaine. Rev. Suisse Agric., 31, 17-22.

Bugaud C., Buchin S., Hauwuy A., Coulon J.B. 2002. Texture et flaveur du fromage selon la nature du pâturage : cas du fromage d'Abondance. INRA Prod. Anim., 15, 31-36.

Bürgi M., Silbernagel J., Wu J., Kienast F. 2015. Linking ecosystem services with landscape history. Landsc. Ecol., 30, 11-20.

Buttler A., 2014. Grasslands in silvopastoral mountain ecosystems. In: Grassland biodiversity and conservation in a changing world. Mariotte P., Kardol P. (Éds). Nova Science Publ., United Kingdom, 187-218.

Buttler A., Gavazov K., Peringer A., Siehoff S. Mariotte P., Wettstein J.B., Chételat J., Huber R., Gillet F., Spiegelberger T., 2012. Conservation des pâturages boisés du Jura : défis climatiques et agro-politiques. Rech. Agron. Suisse, 3, 346-353.

Calabrese C., Mann S., Dumondel M., 2014 Alpine farming in Switzerland: Discerning a lifestyle-driven labor supply. Rev. Soc. Econ. $72,137-156$.

Carrère P., Dumont B., Cordonnier S., Orth D., Teyssonneyre F., Petit M., 2002. L'exploitation des prairies de montagne peut-elle concilier biodiversité et production fourragère? In: Actes du colloque INRA-ENITAC, Agriculture et produits alimentaires de montagne. ENITAC, Lempdes, 41-46.

Carrère P., Seytre L., Piquet M., Landrieaux J., Riviere J., Chabalier C., Orth D., 2012. Une typologie multifonctionnelle des prairies des systèmes laitiers AOP du Massif central combinant des approches agronomiques et écologiques. Fourrages, 209, 9-21.

Carrère P., Farruggia A., Zapata E., Theau J.P. Valadier C., Pauthenet Y., Granet P., Sipan O., Rugraff G., Arranz J.M., Zapata J., Dupic G., Hulin S., 2015. Valoriser les systèmes d'élevage herbagers par la diversité des services rendus par les prairies à l'échelle de petits territoires en zone fromagère AOP. Renc. Rech. Rumin., 22, 133-136.

Chabrat-Michel S., 2015. Impact des conditions territoriales de la mise en place et la mise en place des démarches collectives de valorisation de la viande en moyenne montagne, Thèse de doctorat (Géographie). Université Blaise-Pascal, Clermont-Ferrand, France, 370p.

Chiffoleau Y., Prévost B., 2012. Les circuits courts, une innovation sociale pour une alimentation durable des territoires, Norois, 224, 7-20.

Collomb M., Bütikofer U., Sieber R., Jeangros B., Bosset J.O., 2002. Correlation between fatty acids in cows'milk fat produced in the Lowlands, Mountains and Highlands of Switzerland and botanical composition of the fodder. Intern. Dairy J., 12, 661-666.

Coppa M., Ferlay A., Chassaing C., Agabriel C., Glasser F., Chilliard Y., Borreani G., Barcarolo R., Baars T., Kusche D., Harstad O.M., Verbič J., Golecký J., Martin B., 2013. Prediction of bulk milk fatty acid composition based on farming practices collected through on-farm surveys. J. Dairy Sci., 96, 4197-4211. 
Davis S.C., Burkle L.A., Cross W.F., Cutting K.A., 2014. The effects of timing of grazing on plant and arthropod communities in highelevation grasslands. PLoS One, 9, e110460

Dervillé M., 2012. Territorialisation du secteur laitier et régimes de concurrence, le cas des montagnes françaises et leur adaptation à l'aprèsquota. Thèse de doctorat en Sciences Économiques et Sociales, AgroParisTech, Paris, France.

Diallo A.B, 2017. Evaluation of the economic impact of geographical indications: three case studies, Thèse, Ecole d'Économie de ClermontFerrand, 128p.

Dumont B., Farruggia A., Garel J.P., Bachelard P., Boitier E., Frain M., 2009. How does grazing intensity influence the diversity of plants and insects in a species-rich upland grassland on basalt soils? Grass Forage. Sci., 64, 92-105.

Dumont B., Carrère P., Ginane C., Farruggia A., Lanore L., Tardif A., Decuq F., Darsonville O., Louault F., 2011. Plant-herbivore interactions affect the initial direction of community changes in an ecosystem manipulation experiment. Basic Appl. Ecol., 12, 187-194.

Dumont B., Rossignol N., Loucougaray G., Carrère P., Chadoeuf J., Fleurance G., Bonis A.F., Gaucherand S., Ginane C., Louault F., Marion B., Mesleard F., Yavercovski N., 2012. When does grazing generate stable vegetation patterns in temperate pastures? Agricult. Ecosys. Environ., 153, 50-56.

Dumont B., Ryschawy J., Duru M., Benoit M., Delaby L., Dourmad J.Y., Méda B., Vollet D., Sabatier R., 2017. Les bouquets de services, un concept clé pour raisonner l'avenir des territoires d'élevage. In : Numéro spécial, L'élevage en Europe : une diversité de services et d'impacts. Dumont B. (Éd). INRA Prod. Anim. 30, 407-422.

Duru M., Donnars C., Rychawy J., Therond O., Dumont D., 2017. La "grange": un cadre conceptuel pour appréhender les bouquets de services rendus par l'élevage dans les territoires. In : Numéro spécial, L'élevage en Europe : une diversité de services et d'impacts. Dumont B. (Éd). INRA Prod. Anim., 30, 273-284.

Farruggia A., Pomiès D., Coppa M., Ferlay A., Verdier-Metz I., Le Morvan A., Bethier A., Pompanon F., Troquier O., Martin B., 2014. Animal performances, pasture biodiversity and dairy product quality: How it works in contrasted mountain grazing systems. Agricult. Ecosys. Environ., 185, 231-244.

Ferlay A., Graulet B., Chilliard Y., 2013. Maîtrise par l'alimentation des teneurs en acides gras et en composes vitaminiques du lait de vache. In : Numéro spécial, La vache et le lait. Faverdin P., Leroux C., Baumont R. (Éds). INRA Prod Anim., 26, 177-92.

Ferrez Y., 2007. Contribution à l'étude phytosociologique des prairies mésophiles de Franche-Comté. Les Nouvelles Archives de la Flore jurassienne, 5, 59-151.

Fischer M., von Felten S., Lauber S., 2012. Surface fourragère dans l'exploitation agricole de base - Paramètre clé de la demande d'estivage. Rech. Agron. Suisse, 3, 194-201.

Franzen M., Nilsson S.G., 2008. How can we preserve and restore species richness of pollinating insects on agricultural land? Ecography, 31, 698-708.
Gellrich M., Baur P., Koch B., Zimmermann N., 2007. Agricultural land abandonment and natural forest re-growth in the Swiss mountains: a spatially explicit economic analysis. Agricult. Ecosys. Environ., 118, 93-108.

Gigon A., 2015. De retour depuis 20 ans, le loup échauffe les esprits. Swissinfo, (19 août). http://www.swiss info.ch/fre/a-prot $\%$ C3\% A9ger-ou-\% C3\% A0-abattre- de-retourdepuis-20-ans--le-loup-\% C3\% A9chauffe-lesesprits/41582522

Gillet F., Mauchamp L., Badot P.M., Mouly A., 2016. Recent changes in mountain grasslands: a vegetation resampling study. Ecol. Evol. http://dx.doi.org/10.1002/ece3.1987, -8, 23332345.

Graulet B., Piquet M., Duriot B., Pradel P., Hulin S., Cornu A., Portelli J., Martin B., Farruggia A., 2012. Variations des teneurs en micronutriments de l'herbe de prairies de moyenne montagne et transfert au lait. Fourrages, 209, 59-68.

Hercule J., Chatellier V., Piet L., Dumont B., Benoit M., Delaby L., Donnars C., Savini I., Dupraz P., 2017. Une typologie pour représenter la diversité des territoires d'élevage en Europe. In : Numéro spécial, L'élevage en Europe : une diversité de services et d'impacts. Dumont B. (Éd). INRA Prod. Anim., 30, 285302.

Herzog F., Oehen B., Raaflaub M., Szerencsits E., 2014. Pourquoi l'économie alpestre n'existe pas : tentative de description. In : Avenir de l'économie alpestre suisse, Faits, analyses et pistes de réflexion du programme de recherche AlpFUTUR. Lauber S., Herzog F.; Seidl I., Böni R., Bürg M., Gmür P., Hofer G., Mann S., Raaflaub M., Schick M., Schneider M., Wunderli R. (Éds). Birmensdorf, Institut fédéral de recherche WSL; Zürich-Reckenholz, Station de recherche Agroscope, 19-35.

Homburger H., Hofer G., 2012. Diversity change of mountain hay meadows in the Swiss Alps. Basic Appl. Ecol., 13, 132-138.

INSEE 2015. La filière laitière du bassin Auvergne-Limousin : des atouts à valoriser, $\mathrm{N}^{\circ} 8,4 \mathrm{p}$.

Jeanneaux P., Perrier-Cornet P., 2011. Stratégie d'élévation des coûts des concurrents pour préserver un système productif agro-alimentaire. Rev. Écon. Indust., 135, 115-132.

Junge X., Hunziker M., 2013. Les fonctions de l'économie alpestre jugées par la population. Rech. Agron. Suisse, 4, 272-279.

Klimek S., Richter gen. Kemmermann A., Hofmann M., Isselstein J., 2007. Plant species richness and composition in managed grasslands: the relative importance of field management and environmental factors. Biol. Conserv., $134,559-570$.

Lamarque P., Meyfroidt P., Nettier B., Lavorel S., 2014. How ecosystem services knowledge and values influence farmers' decision-making. Plos One, 9, e107572.

Laurent G., 2014. L'Aubrac : un modèle agricole à renouveler. Université Lyon 2, 37p.

Leiber F., Kreuzer M., Nigg D., Wettstein H.R., Leo Scheeder M.R., 2005. A study on the causes for the elevated n-3 fatty acids in cows' milk of alpine origin. Lipids, 40, 191-202.
Loiseau P., Louault F.., Lhomme G., 1998. Gestion des écosystèmes pâturés en situations extensives : apports de l'écologie fonctionnelle et perspectives de recherches appliquées en moyenne montagne humide. Ann. Zootech., 47, 395-406

Lucas A., Hulin S., Michel V., Agabriel C., Chamba J.F., Rock E., Coulon J.B., 2006. Relations entre les conditions de production du lait et les teneurs en composés d'intérêt nutritionnel dans le fromage : étude en conditions réelles de production. INRA Prod. Anim., 19, 15-28.

Lüscher A., Mueller-Harvey I., Soussana J.F., Rees R.M., Peyraud J.L., 2014. Potential of legume-based grassland-livestock systems in Europe: a review. Grass Forage Sci., 69, 206-228.

Mack G., Flury C., 2014. Quel est l'effet des nouvelles contributions d'alpage? Rech. Agron. Suisse, 5, 88-95.

Mann S., Calabrese C., 2014. Un lieu de travail particulier. In : Avenir de l'économie alpestre suisse. Faits, analyses et pistes de réflexion du programme de recherche AlpFUTUR. Lauber S., Herzog F., Seidl I., Böni R., Bürgi M., Gmür P., Hofer G., Mann S., Raaflaub M., Schick M., Schneider M., Wunderli R. (Éds). Birmensdorf, Institut fédéral de recherche WSL ; Zürich-Reckenholz, Station de recherche Agroscope, 68-79.

Marini L., Fontana P., Scotton M., Klimek S., 2008. Vascular plant and Orthoptera diversity in relation to grassland management and landscape composition in the European Alps. J. Appl. Ecol., 45, 361-370.

Martin B., Verdier-Metz I., Buchin S., Hurtaud C., Coulon J.B., 2005. How do the nature of forages and pasture diversity influence the sensory quality of dairy livestock products? Anim. Sci., 81, 205-212.

Martin B., Hurtaud C., Graulet B., Ferlay A., Chilliard Y., Coulon J.B., 2009. Herbe et qualités nutritionnelles et organoleptiques des produits laitiers. Fourrages, 199, 291-310.

Mauchamp L., 2014. Biodiversité et gestion des écosystèmes prairiaux en Franche-Comté Sciences agricoles, Université de FrancheComté, 280p.

Michaud D., Jeanneaux P., 2014. Éleveurs et coopératives fruitères de la filière Comté face au changement technologique et économique In : Repenser l'économie rurale. Jeanneaux P., Perrier-Cornet P. (Éds). Éditions Quae, Versailles, France, 80-93.

Michelat D., Vionnet G., Giraudoux P., 2015. Impact des pratiques agricoles sur les communautés d'oiseaux : l'exemple de prairies dans le Haut-Doubs. Nos Oiseaux, 62, 67-90.

Miéville-Ott V., Barbezat V., 2005. Perception du pâturage boisé : résultats d'un sondage effectué au Communal de la Sagne NE. Schweizerische Zeitschrift für Forstwesen, 156, 1-12.

Milberg P., Bergman K.O., Cronvall E., Eriksson Å.I., Glimskar A., Islamovic A., Jonason D., Löfqvist Z., Westerberg L., 2016. Flower abundance and vegetation height as predictors for nectar-feeding insect occurence in Swedish semi-natural grasslands. Agricult. Ecosys. Environ., 230, 47-54. 
Milchunas D.G., Sala O.E., Lauenroth W.K., 1988. A generalized-model of the effects of grazing by large herbivores on grassland community structure. Amer. Nat., 132, 87-106.

Mollard A., Rambonilaza M., Vollet D., 2006. Aménités environnementales et rente territoriale sur un marché de services différenciés : le cas du marché des gîtes ruraux labellisés en France. Rev. Écon. Pol., 116, 251-275.

Mollard A.; Dissart, J.C.; Vollet, D., 2014. Valorisation des aménités et développement territorial, les cas des PNR. VertigO, Hors-série 20. http://dx.doi.org/10.4000/vertigo. 15286

Monnet J.C., Berodier F., Badot P.M., 2000. Characterization and localization of a cheese georegion using edaphic criteria (Jura Mountains, France). J. Dairy Sci., 83, 1692-1704

Note P., Michelin Y., 2014. Impact des paysages sur les dynamiques de pullulations de campagnols terrestres dans le massif central, Fourrages, 220, 311-318.

OFAG, 2014. Rapport Agricole 2014. Berne, Suisse: Office fédéral de l'agriculture (OFAG), 320 p.

Pauthenet Y., Roumet J.P., Neyroz A., 1994. Influence de la fertilisation azotée sur la végétation de prairies de fauche en vallée d'Aoste (Italie). Fourrages, 139, 375-378.

Perrier-Cornet P., 1990. Les filières régionales de qualité dans l'agro-alimentaire. Étude comparative dans le secteur laitier en FrancheComté, Emilie Romagne et Auvergne. Écon. Rur., 27-33.

Peter M., Edwards P.J., Jeanneret P., Kampmann D., Lüscher A., 2008. Changes over three decades in the floristic composition of fertile permanent grasslands in the Swiss Alps. Agricult. Écosys. Environ., 125, 204-212.

Peter M., Gigon A., Edwards P.J.L., Andreas, 2009. Changes over three decades in the floristic composition of nutrient-poor grasslands in the Swiss Alps. Biodiv. Conserv., 18, 547-567.
Petit S., Fleur P., Vansteelant J.Y., 2005 Agriculture, prairies de fauche et environnement dans le Massif Jurassien - Outil de diagnostic et conseil. Guide technique: PNR Haut-Jura, Guide Technique, 48p.

Pires J.A., Chilliard Y., Delavaud C., Rouel J., Pomies D., Blanc F., 2015. Physiological adaptations and ovarian cyclicity of Holstein and Montbeliarde cows under two low-input production systems. Animal, 9, 1986-1995.

Ravetto Enri S., Probo M., Farruggia A., Lanore L., Blanchetete A., Dumont B., 2017. A biodiversity-friendly rotational grazing system enhancing flower-visiting insect assemblages while maintaining animal and grassland productivity. Agricult. Ecosys. Environ., 241, 1-10.

Ricard D., 1994, Les montagnes fromagères françaises, thèse de géographie, Université Blaise-Pascal, 496p.

Ricard D., 2013, Permanences et mutation dans les AOC fromagères: Quelques éléments à propos des AOC auvergnates, Revue de la Haute Auvergne, Tome 74, 496-514.

Riedel S., Walter T., Herzog F., 2012 Switzerland. In : High Nature Value Farming in Europe. Oppermann R., Beaufoy G., Jones G (Éds). Germany: Verlag regionalkultur, 420-433.

Ryschawy J., Tichit M., Bertrand S., Allaire G., Plantureux S., Aznar O., Perrot C., Guinot C., Josien E., Lasseur J., Aubert C., Tchakerian E., Disenhaus C., 2015. Comment évaluer les services rendus par l'élevage? Une approche méthodologique sur le cas de la France. INRA Prod Anim., 28, 23-37.

Ryschawy J., Benoit M., Hostiou N., Delfosse C., 2017. Quelles concurrences et synergies entre cultures et élevage dans les territoires de polyculture-élevage? In : Numéro spécial, L'élevage en Europe : une diversité de services et d'impacts. Dumont B. (Éd). INRA Prod. Anim., 30, 363-380.

Seidl I., Böni R., Junge X., Landolt G., Schüpbach B., 2014. Économie alpestre - plus qu'un objet promotionnel pour la société. In : Avenir de 1'économie alpestre suisse. Faits, analyses et pistes de réflexion du programme de recherche AlpFUTUR. Lauber S., Herzog F., Seidl I., Böni R., Bürgi M., Gmür P., Hofer G., Mann S., Raaflaub M., Schick M., Schneider M., Wunderli R. (Éds). Birmensdorf, Institut fédéral de recherche WSL ; ZürichReckenholz, Station de recherche Agroscope, 136-149.

Tasser E., Walde J., Tappeiner U., Teutsch A., Noggler W., 2007. Land-use changes and natural reforestation in the Eastern Central Alps. Agricult. Ecosys. Environ., 118, 115-129.

Tenz R., Elmer R., Huguenin-Elie O., Lüscher A., 2010. Effets de la fumure sur une pelouse à nard raide. Rech. Agron. Suisse, 1, 176-183.

Theau J.P., Chabalier C., Piquet M., Cayre P., Delmas B., Violleau S., Farruggia A., 2012. Construire des outils en partenariat entre Recherche et Développement. Le diagnostic des pratiques fourragères en zone fromagère AOP du Massif central. Fourrages, 209, 69-78.

Verdier-Metz I., Martin B., Pradel P., Albouy H., Hulin S., Montel M.C., Coulon J.B., 2005. Effect of grass-silage vs. hay diet on the characteristics of cheese: interactions with the cheese model. Lait, 85, 469-480.

Vollet D., 2014. Introduction : les enjeux environnementaux au cœur du développement territorial. VertigO, Hors-série, 20. http://dx.doi.org/ $10.4000 /$ vertigo. 15234

Werder C., 2012. Pertes de moutons durant l'estivage. Forum Kleinwiederkäuer / Forum petits ruminants, $10,6-10$

Willisch C., 2014. Protection des troupeaux dans le nord-ouest des Préalpes, 2009-2013. Forum Kleinwiederkäuer / Forum petits ruminants, $1 / 2,9-11$.

You G. 2015 Contractualisation et modes de coordination dans la filière laitière, Écon. Rurale, $345,87-100$.

\section{Résumé}

Les zones herbagères de moyenne et de haute montagne fournissent un bouquet de services équilibré qui comprend des services d'approvisionnement (produits de qualité et disposant d'une bonne image auprès des consommateurs), des services environnementaux et de vitalité territoriale. Afin d'expliciter les déterminants de cette diversité, quatre études de cas ont été retenues à des échelles géographiques différentes : AOP Cantal et Laguiole dans le Massif central, région de Franche-Comté, alpages suisses. Une même grille d'analyse a été appliquée à chacune de ces études de cas. Le contexte qui conduit à la situation actuelle est présenté dans une perspective historique, avant que soient analysés leurs principaux services d'approvisionnement, environnementaux et de vitalité territoriale. Chaque étude de cas est synthétisée par une formalisation inspirée d'une analyse en termes de système socio-écologique. Nous discutons par la suite des leviers permettant de mieux concilier les performances productives, environnementales et sociales de l'élevage dans ces territoires. Un levier biotechnique majeur est la recherche d'autonomie fourragère qui permet de réduire les coûts de production tout en bénéficiant à la qualité nutritionnelle des laits et des fromages. Il existe par ailleurs des liens forts entre la nature de la gouvernance des filières et le bouquet de services fournis. Une gouvernance territoriale de filières crée de la valeur en valorisant les ressources locales, et en régulant le système de production par des organisations dédiées ; elle bénéficie conjointement aux services de vitalité territoriale et de qualité de l'environnement. A l'inverse, une gouvernance sectorielle apparait moins favorable. Le développement de formes hybrides de gouvernance (par exemple alimentaire territoriale) constitue l'un des leviers importants. 


\begin{abstract}
Bundles of services in grassland-based cheese production systems from preserved environment areas

Upland grassland areas can provide a balanced bundle of services, including provisioning services (high-quality products with a positive image for consumers), environmental services and territorial vitality. In order to analyze drivers of this diversity, four case studies were analyzed: Cantal and Laguiole production areas in the French Massif central, Franche-Comté region, and Swiss alpine pastures. Before presenting bundles of services in each area, we analyzed the context that led to the current situation from a historical perspective. Each case study was then represented using a simplified formalization derived from an analysis in terms of socio-ecological system. We finally discuss options for reconciling the productive, environmental and social performances of grassland-based livestock farming systems. It appears crucial to increase the fodder autonomy of dairy farms in order to reduce production costs and to increase the nutritional quality of milk and cheese. There are also strong links between the nature of the governance of value chains and the nature of the services provided. Territorial governance forms create more value by enhancing the use of local resources and by regulating production by dedicated professional organizations; they also appear to benefit environmental services and territorial vitality. Conversely, sectoral governance appears to be less favorable. Development of a hybrid form of governance (e.g. territorial alimentary) is one important option.
\end{abstract}

VOLLET D., HUGUENIN-ELIE O., MARTIN B., DUMONT B., 2017. La diversité des services rendus par les territoires d'élevage herbagers fournissant des produits de qualité dans des environnements préservés. In : Numéro spécial, L'élevage en Europe : une diversité de services et d'impacts. Dumont B. (Éd). INRA Prod. Anim., 30, 333-350. 
